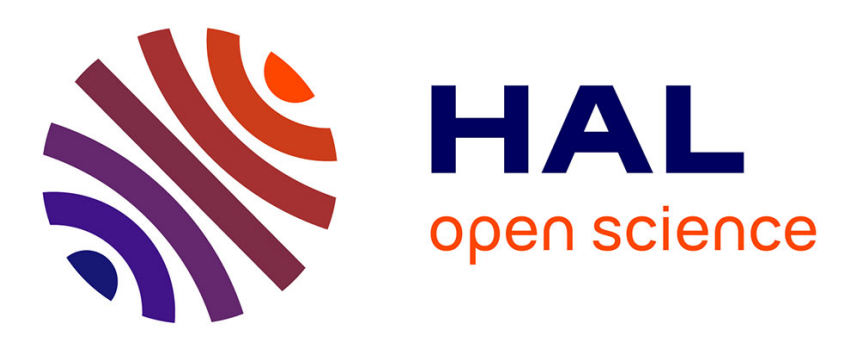

\title{
Waves, modes and properties with a major impact on dynamic pantograph-catenary interaction
}

\author{
Olivier Vo Van, Jean-Pierre Massat, Etienne Balmes
}

\section{To cite this version:}

Olivier Vo Van, Jean-Pierre Massat, Etienne Balmes. Waves, modes and properties with a major impact on dynamic pantograph-catenary interaction. Journal of Sound and Vibration, 2017, 402 (août 2017), pp.51-69. 10.1016/j.jsv.2017.05.008 . hal-01534188

\section{HAL Id: hal-01534188 \\ https://hal.science/hal-01534188}

Submitted on 7 Jun 2017

HAL is a multi-disciplinary open access archive for the deposit and dissemination of scientific research documents, whether they are published or not. The documents may come from teaching and research institutions in France or abroad, or from public or private research centers.
L'archive ouverte pluridisciplinaire HAL, est destinée au dépôt et à la diffusion de documents scientifiques de niveau recherche, publiés ou non, émanant des établissements d'enseignement et de recherche français ou étrangers, des laboratoires publics ou privés. 


\title{
Waves, modes and properties with a major impact on dynamic pantograph-catenary interaction
}

\author{
Olivier Vo Van ${ }^{\mathrm{a}, \mathrm{b}, 1, *}$, Jean-Pierre Massat ${ }^{\mathrm{c}}$, Etienne Balmes $^{\mathrm{a}, * *}$ \\ ${ }^{a}$ Arts et Metiers ParisTech, PIMM, 151 Boulevard de l'hopital, 75013, Paris, France \\ ${ }^{b}$ SNCF Innovation 83 Recherche, 40 Avenue des Terroirs de France, 75012, Paris, France \\ ${ }^{c}$ SNCF Ingénierie 83 Projets, 6 Avenue François Mitterrand, 93574 la Plaine St Denis Cedex, France
}

\begin{abstract}
Understanding the dynamic behavior of the pantograph-catenary system is crucial for design improvement, but many factors influence the contact force, which is the main design objective. To give a proper understanding of dynamic characteristics, the paper uses a combination of mass drop tests on a catenary, analytic models and parametric finite element model simulations allowing a fine analysis of the influence of train speed. The first contributor to contact force variations is the geometry of the catenary under gravity loading. This parameter is however shown to be insufficient to explain higher frequency effects. The second contributor is the propagation of waves in the contact and messenger wires. The influence of wave dispersion is first demonstrated, which emphasizes the importance of considering the bending stiffness. Wave compensation by droppers and reflections at the mast are then shown to be important. Characteristic times associated with wave group velocities are finally used to explain the series of harmonic contributions visible in spectra in the catenary and pantograph frames. Finally, modes are shown to play a role particularly when their frequencies coincide with other contributions. The notion of mode groups, associated wave velocities and relevant design variables are discussed. Several observations pave the way for future work on catenary design.
\end{abstract}

Keywords: Pantograph-catenary dynamics, wave propagation, frequency coincidences, catenary geometry

\section{Introduction}

Catenaries are the main means of transmitting electric current to trains. These cable assemblies are fairly large, combining weakly coupled spans, typically around $54 \mathrm{~m}$ long, into sections of about $1 \mathrm{~km}$. The classical composition of a high speed catenary is displayed in figure 1. Current is collected by pantographs interacting with the contact wire and the contact force is used as the main quantity of interest for the validation of current collection quality. This industrial system, thus combines a range of interesting dynamic properties: influence of geometry, waves generated by moving loads, fixed and moving frame dynamics, groups of modes.

The impact of catenary geometry was studied through sensitivity analysis of the pre-sag [1] or other control parameters [2]. Wave propagation in the cables is also a well-studied problem as it is the main limit to the train velocity. The critical velocities have been analysed by Náprstek [3] for the case of a moving load under a string on a Winkler suspension. Its impact on dynamic has been studied analytically [4] and experimentally [5], with differing results with respect to the characteristic velocity of waves in the catenary.

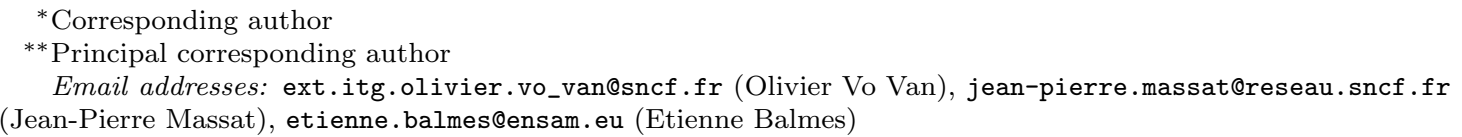




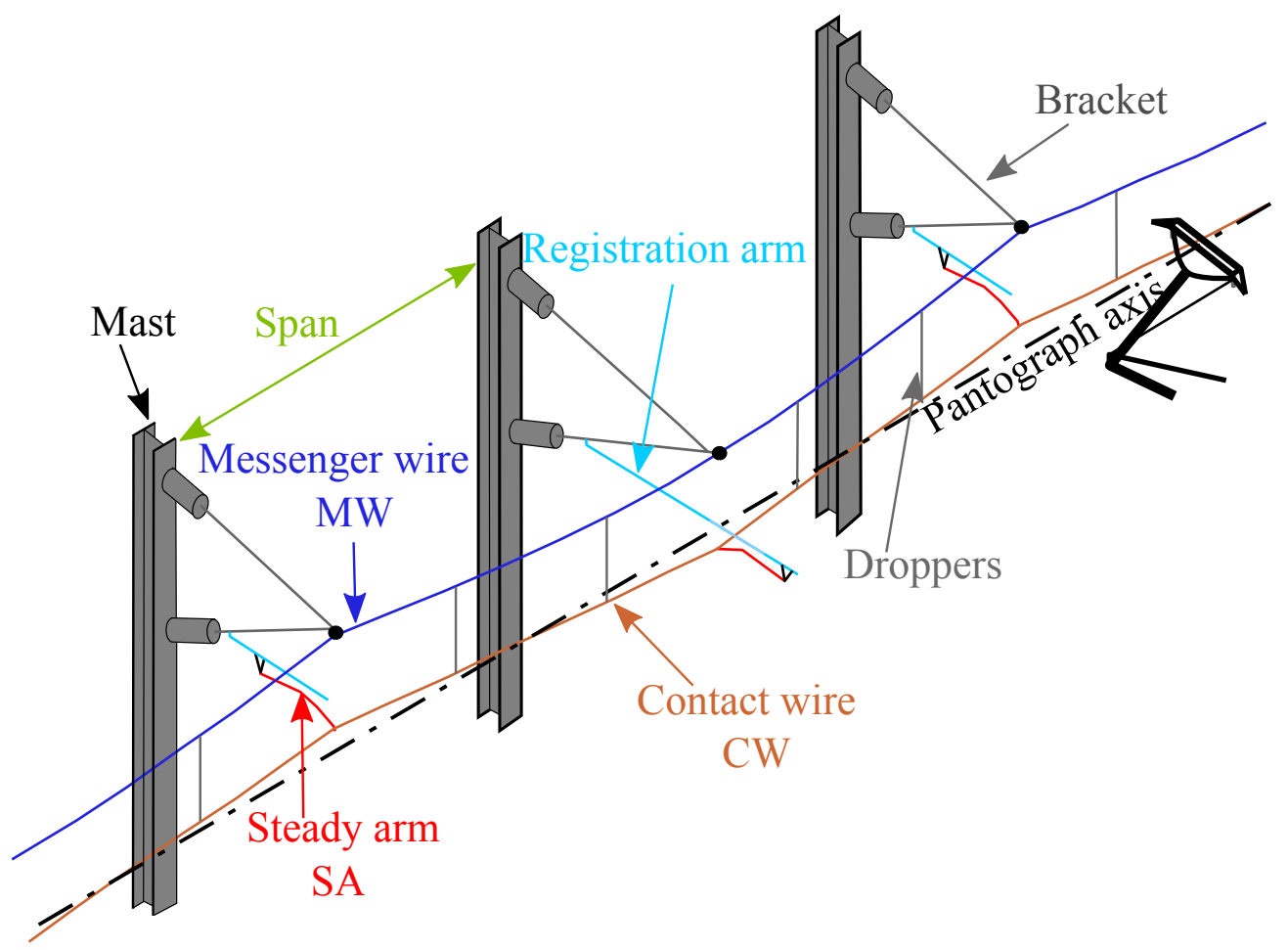

Figure 1: Composition of a typical $25 k \mathrm{~V}$ catenary, a contact wire (CW) suspended to messenger wire (MW) by droppers

To open the path for industrial uses in maintenance such as wear $[6,7,8]$ or fatigue studies [9], design by optimisation $[10,11]$ or sensitivity analysis $[12,13,14,15]$, various numerical models have been developed and were compared in a benchmark study [16] demonstrating that predictive models exist. The non-linear behaviour of the catenary has a significant impact $[17,18]$, which is why Finite Element Models (FEM) are prefered. Good understanding of factors influencing contact force fluctuations is however not widespread and this paper, using a combination of tests, analytic models and large scale FEM simulations, seeks to fill this gap.

After a description of the considered test, model and simulation configurations in section 2, section 3 addresses the impact of the geometry of the contact wire (CW) which is deformed by gravity. An analytic model is used to show contact force contributions obtained by the convolution of pantograph dynamic impedance and geometry profile. For high speeds, this is shown to be a major contributor to contact force fluctuations.

Since geometry leaves a number of aspects unexplained, wave propagation is considered in section 4 . Tests are used to demonstrate wave speeds and the level of wave dispersion found in reality. An analytic model is then used to analyse reflections on droppers (see figure $2 b$ ), which is the first effect seen in mass drop tests. As masts represent stiff points in the catenary, wave propagation in their vicinity is fairly critical and a second analytical model is used to highlight the main effects.

To analyse the effect of waves on the coupled pantograph/catenary system, section 5 considers time of flight associated with wave fronts generated by the pantograph in both the fixed frame and that moving with the pantograph.

Finally section 6 addresses modes. The weakly coupled spans are shown to generate group of modes, whose frequencies are notably influenced by tensions in the CW and messenger wires (MW which is the supporting wire). This is in particular used to justify that the wave velocity relevant when analysing responses is the mean of velocities in both wires. The section finally shows effects associated with the 
interaction of multiple pantographs.

\section{Configurations for test and FEM simulations}

While tests described in section 2.1 are original, the amount of available information is low and less flexible than the numerical model detailed in section 2.2. Simulations will thus be used to understand physical phenomena and measurements to validate results.

\subsection{Measurements}

Dynamic measurements are generally collected during certification tests of rolling stock or catenary. Only contact force between the pantograph and the catenary and uplift of the CW under a steady arm are then measured. These data are always localised in space and correspond to a coupled system at a single train velocity.

For all these reasons, tests were led on a catenary alone. The tests consisted in mass drops at different points of the catenary while measuring accelerations with four tri-axial accelerometers of type 8395-A010 from Kistler which have a sensitivity of $400 \mathrm{mV} / \mathrm{g}$ and a frequency response in the range $[0-1] \mathrm{kHz}$. As the noise levels were quite low, integration to compute velocities was possible and the associated results will be used. Figure $2 \mathrm{a}$ shows a picture of a steady arm (SA) and figure $2 \mathrm{~b}$ one accelerometer placed at the bottom of a dropper. Two test configurations are shown in figures $2 \mathrm{c}$ and $2 \mathrm{~d}$ with sensors positions (red) and the positions of the masses dropped (blue). Tests were led inside a span (2c) to observe reflections and transmissions on droppers, or in the span without any dropper $(2 \mathrm{~d})$ to observe wave propagation and reflection/transmission on a mass (black) when one is added.

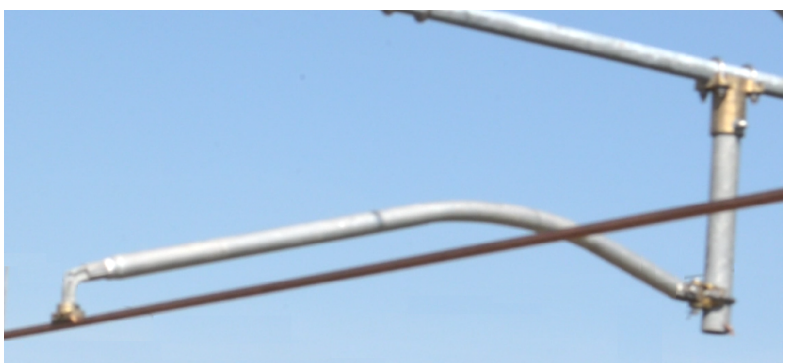

(a)

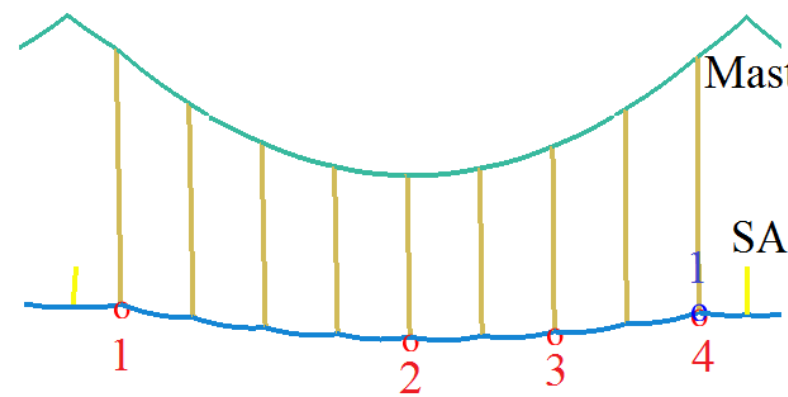

(c)

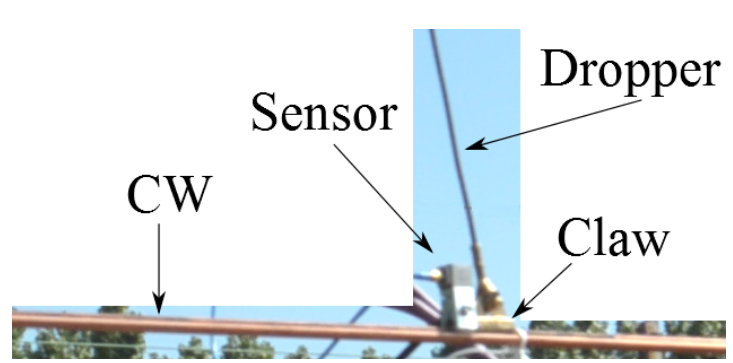

(b)

Figure 2: (a) Picture of a steady arm; (b) Picture of an accelerometer placed next to a dropper claw on the contact wire; Mass drop configurations inside a span (c) with droppers; (d) without dropper 


\subsection{FEM simulations}

The catenary is generally modelled using FEM. Droppers are modelled as bars with non-linear stiffness to represent the loss of stiffness in compression. Steady and registration arms are represented using bars or beams with pin flags thus allowing pivot motion at their ends. The various claws (for droppers, arms or junctions) are modelled using concentrated masses. Figure 3a shows the FEM representing the catenary on which measurements of section 2.1 were led. The pantograph of figure $3 \mathrm{~b}$ is modelled by three lumped masses connected by springs representing the first few modes. Figure 6 a shows this pantograph model with the contact between pantograph and catenary modelled as a contact stiffness.

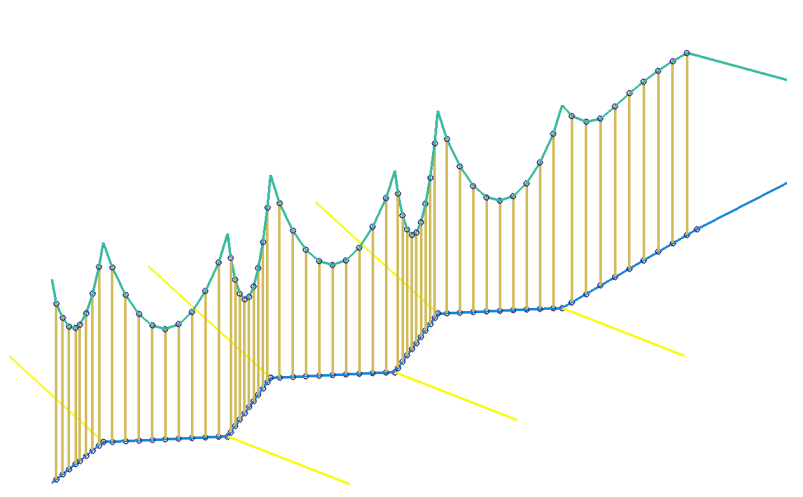

(a)

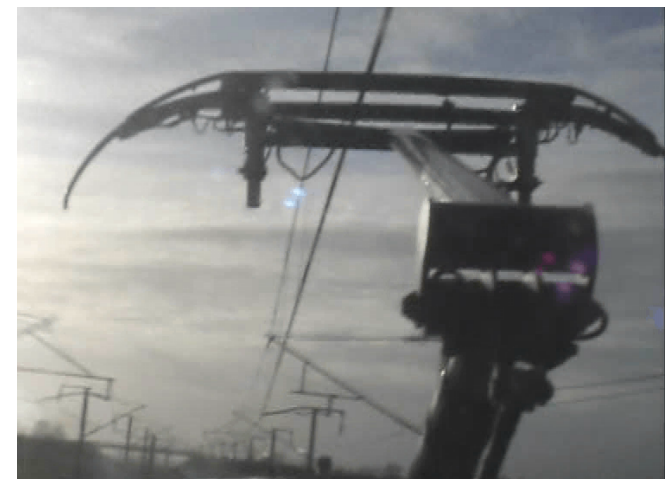

(b)

Figure 3: (a) Catenary FEM; (b) Picture of a pantograph

The OSCAR software package, used in this work, has been under continuous development by SNCF and SDTools since 2003. The simulation software is described in [19,20]. Recent improvements in computation capacity enable simulation of contact forces and catenary displacements for a large range of velocities which will be used in many illustrations of this work.

A sample of a $20 \mathrm{~Hz}$-filtered contact force simulated with a train passing at $90 \mathrm{~km} / \mathrm{h}$ and $320 \mathrm{~km} / \mathrm{h}$ in a nominal configuration along five spans is shown in figure 4 . It is filtered at $20 \mathrm{~Hz}$ since this is the traditional bandwidth of estimated forces in tests. Passages at masts (or under steady arms) are indicated with vertical dotted lines. The first obvious observation is that, signals being plotted against the pantograph position, the filtering frequency of $20 \mathrm{~Hz}$ in the temporal domain gives a totally different spatial filtering if the pantograph velocity is changed. A spatial filtering is thus more appropriate to compare contact force at different speed. 


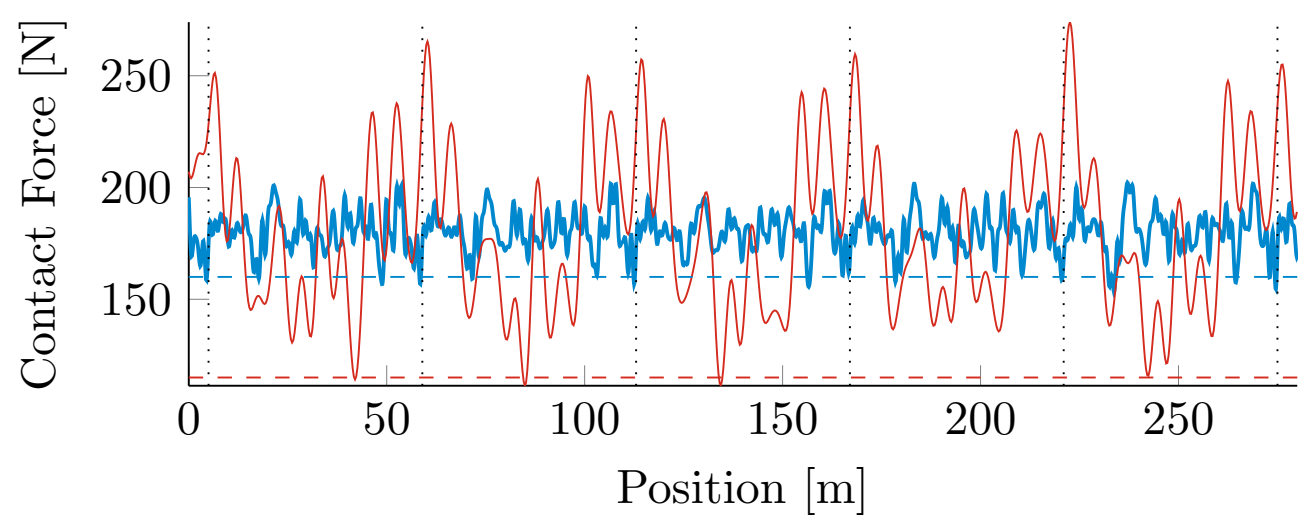

Figure 4: $20 \mathrm{~Hz}$-filtered contact force simulated at $90 \mathrm{~km} / \mathrm{h}$ (thick blue) and $320 \mathrm{~km} / \mathrm{h}$ (thin red); corresponding value of $F_{m}-3 \sigma$ in dashed lines

The current collection quality is generally assessed by two criteria. The first is on the coefficient of variation,

$$
\frac{\sigma}{F_{m}}<0.3
$$

with $\sigma$ the standard deviation of the contact force and $F_{m}$ its mean. This criterion is almost equivalent to $F_{m}-3 \sigma>0$. It ensures that if the contact force statistic distribution is Gaussian, the contact is lost less than $1 \%$ of the time and thus that electric wear due to arcs is limited. The limit $F_{m}-3 \sigma$ is indicated as dashed lines in figure 4.

The second is on the mean contact force that has to be as small as possible to reduce the mechanical wear.

\section{A contact moving on a deformed geometry}

Due to gravity, the catenary is never perfectly flat. The first phenomenon analysed in this section is the dynamic response of the pantograph to the static catenary profile, which corresponds to a fixed catenary assumption. The pantograph-catenary system is known to be coupled, but this section will show that significant contributions can be analysed even with coupling ignored, thus showing the relevance of uncoupled analysis. An analytical model is developed in section 3.1 and results are then compared with simulations in section 3.2 .

\subsection{Analytical model of a pantograph moving on the static catenary geometry}

The geometry of the catenary corresponds to the height of the CW along the catenary, which is defined in the direction $\vec{x}$. Figure 5a shows a sample geometry along one span. Steady arms are indicated by vertical solid lines and droppers by vertical dotted lines. The section is chosen periodic so that this geometry can be characterized by its Fourier transform in the spatial domain, $H_{\mathrm{CW}}(k)$.

A pantograph is passing under the catenary at a speed of $v_{\text {panto }}$. The pantograph sees the height of the $\mathrm{CW}$ vary with a velocity $V_{\mathrm{CW}}(k)=\mathrm{i} k H_{\mathrm{CW}}(k)$ in the spatial Fourier domain. The angular frequency $\omega$ in the temporal Fourier domain is linked to $k$ by the velocity of the pantograph $v_{\text {panto }}$,

$$
\omega=v_{\text {panto }} \cdot k,
$$

which leads to the relation $V_{\mathrm{CW}}(\omega)=v_{\text {panto }} V_{\mathrm{CW}}(k)$. 
The amplitude spectrum of $V_{\mathrm{CW}}(k)$ shown in figure $5 \mathrm{~b}$ is clearly composed of a periodic part with a fundamental spatial frequency $k_{\text {span }}$ defined by

$$
k_{\mathrm{span}}=\frac{1}{d_{\mathrm{span}}}
$$

where $d_{\text {span }}$ is the length of one span, and a non periodic part at a significantly lower level.
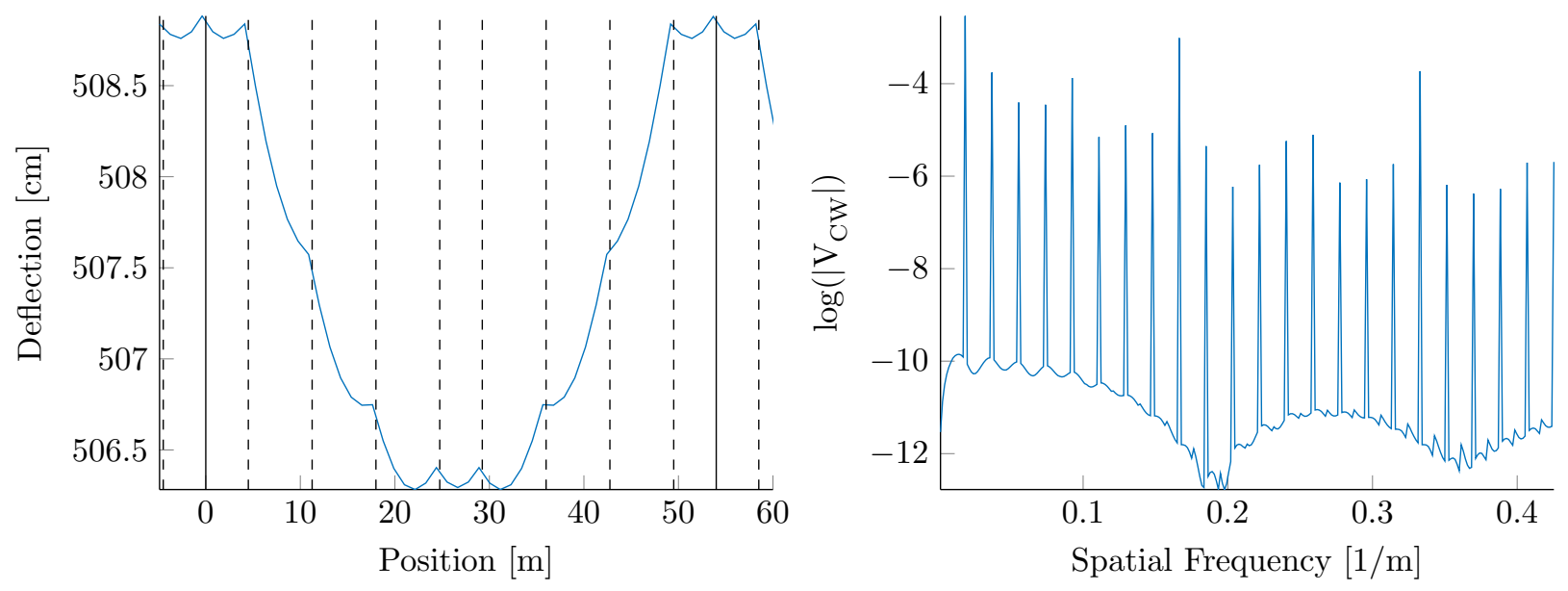

(a)

(b)

Figure 5: (a) Height of the contact wire along one span, $h_{\mathrm{CW}}(x)$; (b) Amplitude spectrum of the vertical spatial velocity of the CW, $V_{\mathrm{CW}}$.

As illustrated by figure $6 \mathrm{a}$, the base lumped mass pantograph model is a 3 mass/spring/damper $\left(m_{i}, d_{i}, k_{i}\right)$ system linked to the CW by a contact stiffness $k_{c}$ typically assumed to be constant. To analyse the effect of geometry, the wire is assumed to be fixed in its initial static position. The output wave velocities generated in front and behind the pantograph, respectively $V_{t+\text {,panto }}$ and $V_{t-\text {,panto }}$ and the reflected wave $V_{r}$ will only be considered in section 4 and thus assumed null for now. The mean force $F_{0}$ and the velocity $V_{\mathrm{CW}}$ are considered inputs and the output of interest is the contact force due to geometry $F_{\text {geom }}$. 


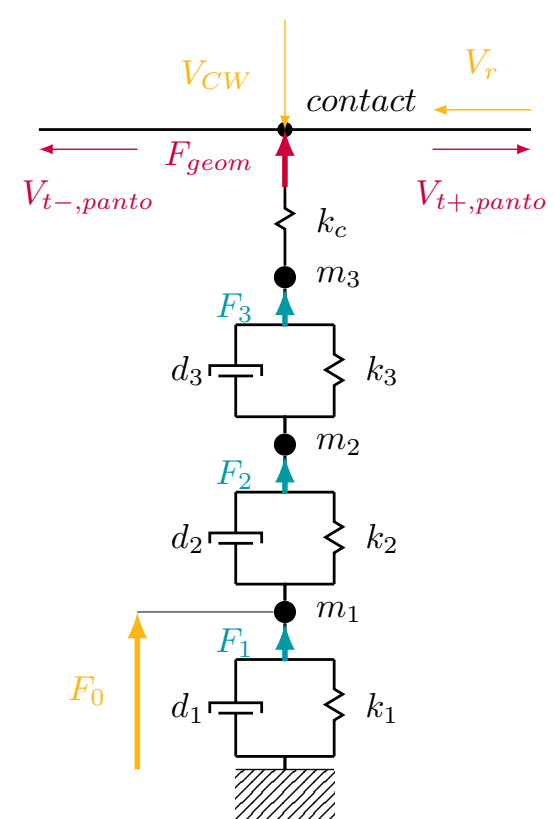

(a)

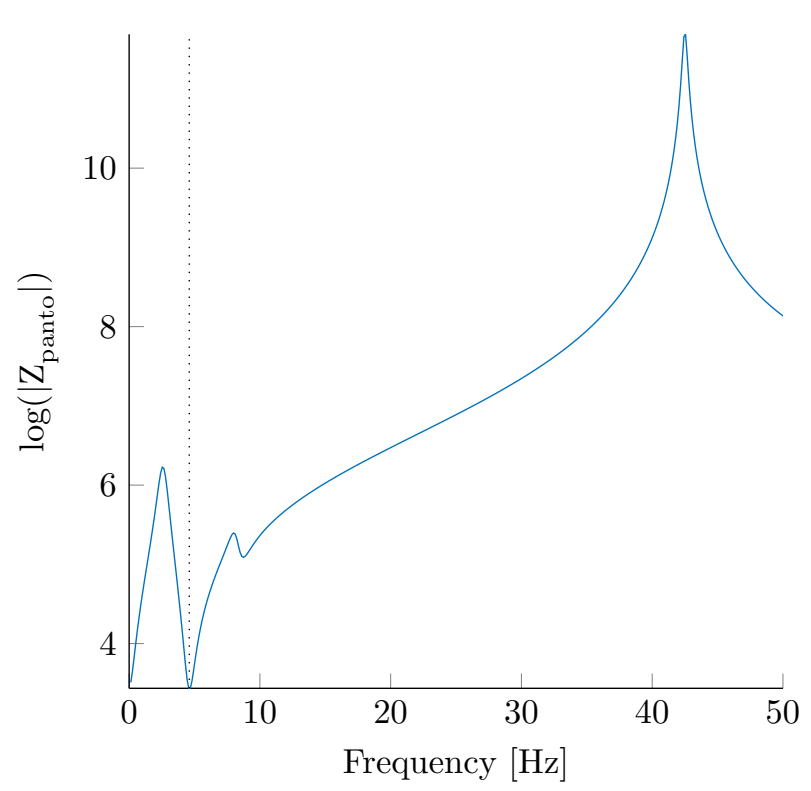

(b)

Figure 6: (a) Pantograph model with the contact wire; (b) Dynamic impedance of the pantograph $Z_{\text {panto }}$.

For each damper-spring cell, the relation between force and velocity is given by

$$
\Delta F_{j}(\omega)=\Delta V_{j}(\omega) \cdot\left(d_{j}+\frac{k_{j}}{\mathrm{i} \omega}\right)
$$

Equilibrium at the masses, $m_{1}, m_{2}$ and $m_{3}$ give the system equations

$$
\begin{aligned}
& {\left[\left[\begin{array}{ccc}
m_{1} & 0 & 0 \\
0 & m_{2} & 0 \\
0 & 0 & m_{3}
\end{array}\right] \mathrm{i} \omega_{p}+\left[\begin{array}{ccc}
d_{1}+d_{2} & -d_{2} & 0 \\
-d_{2} & d_{2}+d_{3} & -d_{3} \\
0 & -d_{3} & d_{3}
\end{array}\right]+\right.} \\
& \left.\left[\begin{array}{ccc}
k_{1}+k_{2} & -k_{2} & 0 \\
-k_{2} & k_{2}+k_{3} & -k_{3} \\
0 & -k_{3} & k_{3}+k_{c}
\end{array}\right] \frac{1}{\mathrm{i} \omega}\right]\left\{\begin{array}{l}
V_{1} \\
V_{2} \\
V_{3}
\end{array}\right\}=\left[\begin{array}{c}
F_{0} \\
0 \\
\frac{k_{c}}{\mathrm{i} \omega} \cdot V_{\mathrm{CW}, 0}
\end{array}\right]
\end{aligned}
$$

$F_{\text {geom }}$, the contact force resulting from contact point motion on the geometry, is obtained from the force velocity relation of the contact spring,

$$
F_{\text {geom }}(\omega)=\frac{k_{c}}{\mathrm{i} \omega}\left(V_{\mathrm{CW}}(\omega)-V_{3}\right) .
$$

Denoting $\mathbf{A}$ the left matrix in (5), $\mathbf{A}^{-1}$ can be computed and expressions of outputs evaluated

$$
\begin{array}{cl}
V_{3}(\omega)=\left(\mathbf{A}^{-1}\right)_{3,1} \cdot F_{0}+\left(\mathbf{A}^{-1}\right)_{3,3} \cdot \frac{k_{c}}{\mathrm{i} \omega} V_{\mathrm{CW}}(\omega) \\
F_{\text {geom }}(\omega)=\frac{k_{c}}{\mathrm{i} \omega}\left(1-\left(\mathbf{A}^{-1}\right)_{3,3} \cdot \frac{k_{c}}{\mathrm{i} \omega}\right) V_{\mathrm{CW}}(\omega) & +\frac{k_{c}}{\mathrm{i} \omega}\left(\mathbf{A}^{-1}\right)_{3,1} \cdot F_{0} \\
=Z_{\text {panto }}(\omega) V_{\mathrm{CW}}(\omega) & +F_{m} \\
=v_{\text {panto }} Z_{\text {panto }}(\omega) V_{\mathrm{CW}}(k) & +F_{m}
\end{array}
$$


As $F_{m}$ is null for any $\omega \neq 0, F_{\text {geom }}$ is the product of $Z_{\text {panto }}$ and $V_{\mathrm{CW}}$ in the frequency domain and a convolution in the time domain. Figure $6 \mathrm{~b}$ shows the dynamic impedance $Z_{\text {panto }}(\omega)$ in the pantograph frame and figure $5 \mathrm{~b}$, the spatial Fourier transform of the geometry $V_{\mathrm{CW}}(k)$. The resulting force $F_{\text {geom }}$ is thus a convolution of these two spectra.

\subsection{Sample results}

The analytical model of $F_{\text {geom }}$ can be compared to simulations. The $F_{\text {geom }}$ force can be displayed against time or spatial frequency and depends on velocity $v_{\text {panto }}$.

The catenary section, used as illustration for this section, is composed of fourteen 54m-length spans with a total length of $1135 \mathrm{~m}$. The typical computation time for dynamic simulations of a model with 9000 degree of freedom varies from 7 , for high velocities, to 25 minutes for low velocities. 230 simulations were needed for the map in Figure $7 \mathrm{~b}$ and made possible by recent improvement of computational capacity. These new numerical capabilities were a key needed to build the analysis proposed in this paper.

Figure 7 compares $F_{\text {geom }}$ to the Fourier transform of the dynamic contact force $F_{c}$ computed for different values of pantograph velocity. The velocity is normalized by the velocity of waves in cables, $v_{\phi}$. This wave velocity will be defined more precisely in section 6.3 .

$$
\beta=\frac{v_{\text {panto }}}{v_{\phi}}
$$

It is clear that the peaks defined by $V_{\mathrm{CW}}$ can be observed in $F_{c}$ and the amplitudes are quite close. The influence of $Z_{\text {panto }}$ is less clear, but can still be observed as curves from top-left to bottom-right. To highlight this trend, the frequency of the anti-resonance shown as a vertical dotted line in figure $6 \mathrm{~b}$ is used. Since the anti-resonance is fixed in temporal frequency in the moving frame and a spatial frequency is used for the display, equation (2) leads to a spatial frequency $k_{z}=\omega_{z} / v_{\text {panto }}$ which is overlaid as a dotted curve in figure 7 .

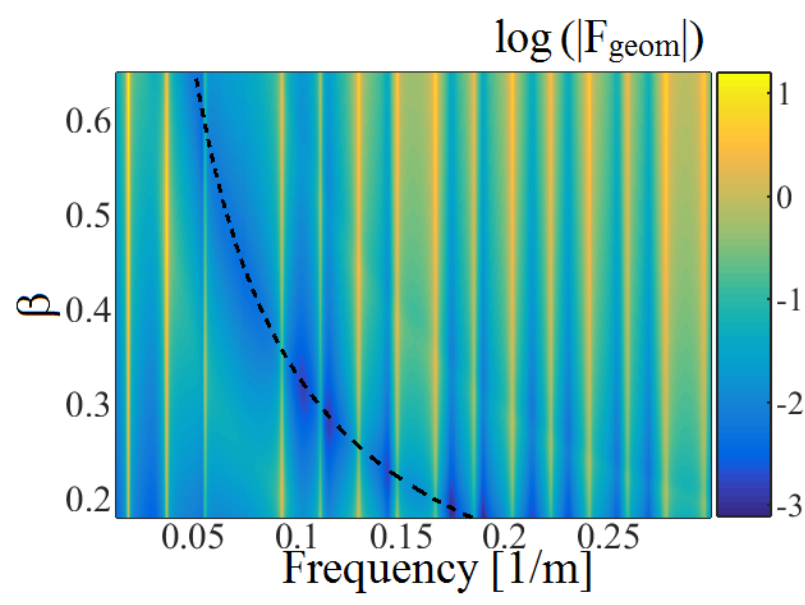

(a)

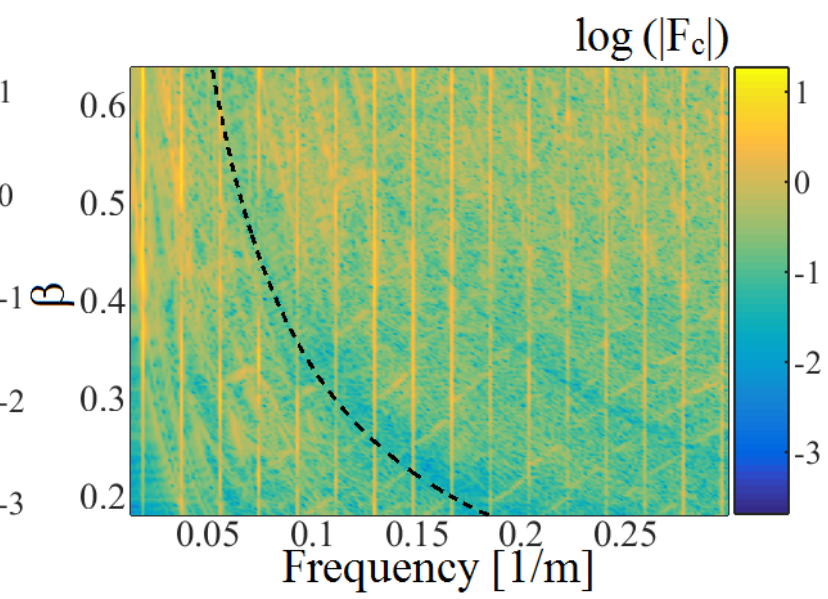

(b)

Figure 7: (a) Analytic contact force due to catenary geometry $F_{\text {geom }}$; (b) Contact force $F_{c}$ computed by dynamic simulations.

To go back to the time domain, the inverse Fourier transform of $F_{\text {geom }}(k)$ can be computed and compared with simulations. Figure 8 shows this comparison for two different speeds, namely $90 \mathrm{~km} / \mathrm{h}$ and $320 \mathrm{~km} / \mathrm{h}$ corresponding to $\beta=0.18$ and $\beta=0.64$. The force fluctuation induced directly by the geometry of the catenary increases with speed and represents the main part of the $20 \mathrm{~Hz}$-filtered contact force at high speed. For low speed, other phenomena cause the contact force to significantly differ from $F_{\text {geom }}$. The catenary dynamic is assumed to be the cause. 

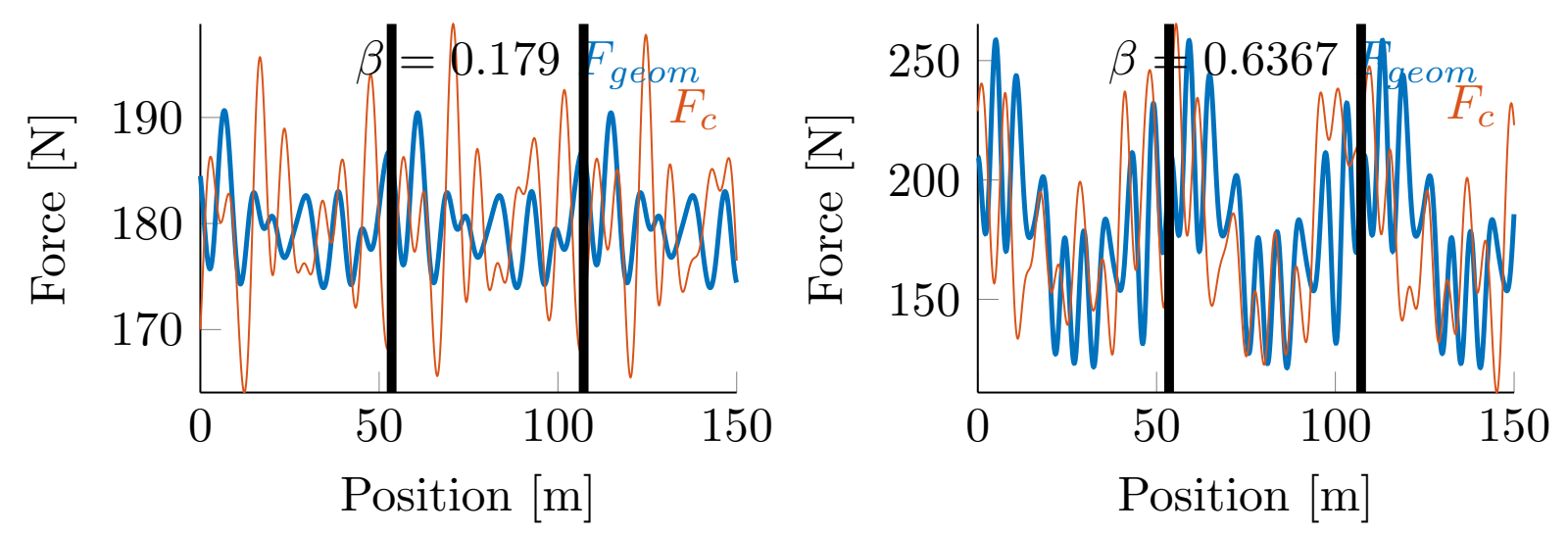

(a)

(b)

Figure 8: Force induced by geometry (thick blue) and simulated dynamic contact force (thin red). (a) $v_{\text {panto }}=90 \mathrm{~km} / \mathrm{h}$; (b) $v_{\text {panto }}=320 \mathrm{~km} / \mathrm{h}$.

When the contact force is observed at higher frequencies, for example using a $70 \mathrm{~Hz}$ low pass filter, the similarity between the force due to geometry $F_{\text {geom }}(x)$ and the dynamic contact force $F_{c}(x)$ is less clear. It thus clearly appear that the current practice of filtering tests at $20 \mathrm{~Hz}$ is not able to capture wave information for high speed trains.

In conclusion, the force due to geometry is a major contributor to low temporal frequency contact force fluctuations particularly at high speeds. This provides an extremely fast meta-model of contact force evolutions that could be used for pantograph or catenary design. The proposed analytical model is limited by the fact that it does not represent wave propagation effects.

\section{Waves propagating in an assembly of tensioned wires}

The second phenomenon that induces contact force fluctuations is the wave propagation in cables of the catenary. Such propagation is known to have a strong impact on pantograph-catenary dynamic interaction [5, $4,3]$ and this section first presents experimental and numerical validation for fixed loads.

Wave dispersion is the first characteristic of a wave guide. Section 4.1 shows that this phenomenon observed in measurement. The string assumption is then used in sections 4.2 and 4.3 to model waves propagation in wires and their reflections and transmissions against droppers, masts and steady arms. This is used to gain insight on velocity changes associated with reflections and transmissions. Tests detailed in [21] indicate a very low damping close to material properties. Since that is low and constant, no study on damping was performed.

\subsection{String vs. beam models, the impact of dispersion}

Considering a beam of Young Modulus $E$, moment of inertia $I$ and linear mass $\mu$ under an axial load $T$, equations of motion for an unconstrained Euler-Bernoulli beam with tension are well known (for example [22]) and take the form

$$
E I \frac{\partial^{4} z}{\partial x^{4}}-T \frac{\partial^{2} z}{\partial x^{2}}+\mu \frac{\partial^{2} z}{\partial t^{2}}=F
$$

where $F$ is the external force applied on the beam.

For the beam model, assuming waves of the form $\mathrm{e}^{\mathrm{i}(k x-\omega t)}$ leads to the dispersion equation

$$
\left(E I k^{4}-\mu \omega^{2}+T k^{2}\right)=0,
$$


relating wave number $k$ and frequency $\omega$.

In analysing waves, quantities of interest are the phase velocity $c_{\phi}$ and the group velocity $c_{g}$ [23] defined here as

$$
\left\{\begin{array}{l}
c_{\phi}=\frac{\omega}{k}=\sqrt{\frac{T+E I k^{2}}{\mu}} \\
c_{g}=\frac{\partial \omega}{\partial k}=\frac{E I k^{2}}{\sqrt{\mu} \sqrt{T+E I k^{2}}}+c_{\phi} .
\end{array}\right.
$$

With the string hypothesis, the bending moment $E I$ is neglected, which leads to the constant wave velocity of strings,

$$
c_{0}=\sqrt{\frac{T}{\mu}} .
$$

The curve of group velocity as a function of frequency is thus a horizontal line in the string case. $c_{0}$ is known as the critical velocity of the wire. This is one of the main limits for the train velocity.

To understand the frequency limit of the string hypothesis, measurements in configuration of figure $2 \mathrm{~d}$ are used. Figure 9a shows the vertical velocity of the CW after a mass drop $20 \mathrm{~m}$ away from the sensor. Oscillations can be observed progressively increasing before the step corresponding to the wave arrival at the sensor. These oscillations are an experimental observation of wave dispersion (or non-constant group velocity). Figure $9 \mathrm{~b}$ shows the evolution of group velocity with frequency. The estimate is based on a spectrogram representing the Fourier transform of a sliding window of the time signal. Rather than displaying amplitude as a function of frequency and time, one builds a velocity axis by dividing the mass drop to sensor distance by the time, thus leading to the figure shown. It clearly appears that the string assumption becomes notably wrong at frequencies above $50 \mathrm{~Hz}$. Since for every speed, propagating waves exist at certain frequencies, keeping bending effects is particularly important for research on critical and super-critical velocities.

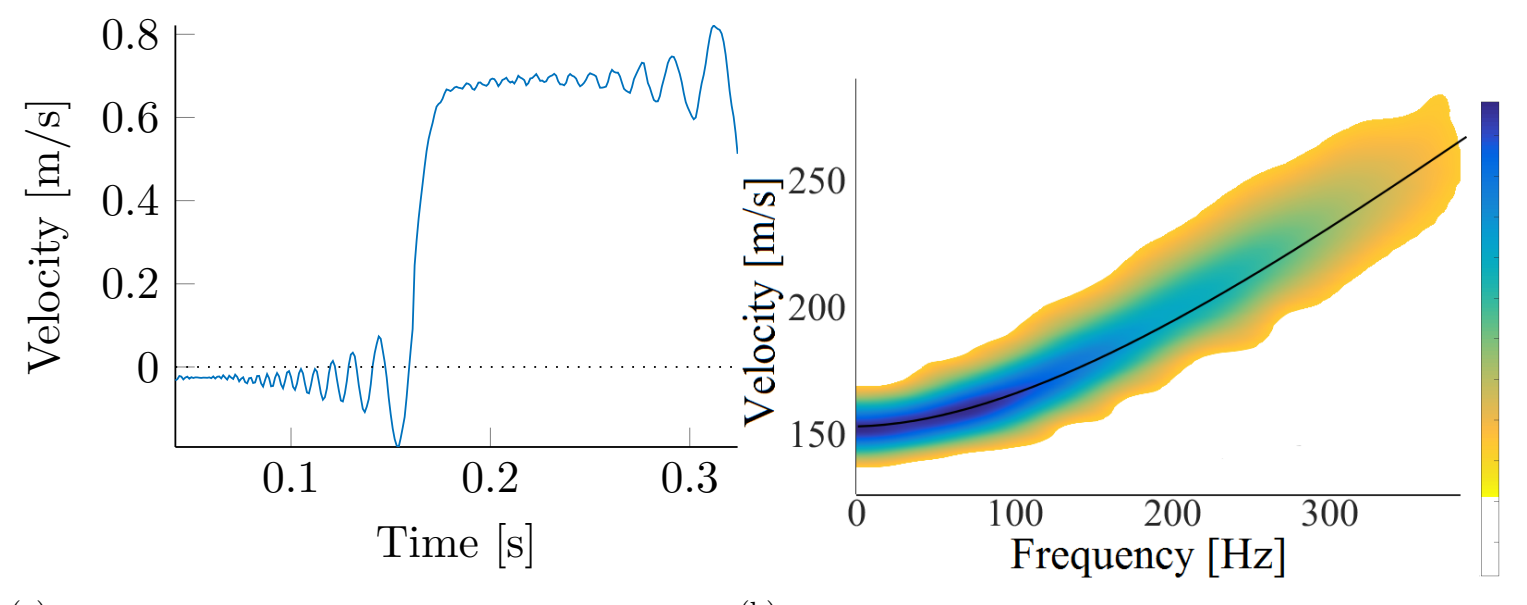

(a)

(b)

Figure 9: Dispersion of wave propagated observed on a measurement after a mass drop 20m away from the sensor; (a) vertical velocity; (b) Evolution of wave velocity with frequency using a spectrogram.

In further sections, test and FEM simulations are affected by dispersion, while analytic models consider strings which give clearer results. The first case analysed is the wave reflection and transmission against a dropper, and the second focuses on the mast area. 


\subsection{Analytic model of wave reflection and transmission at a dropper}

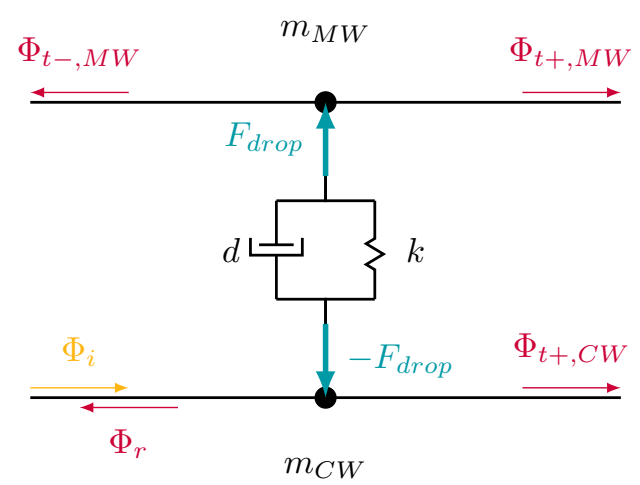

Figure 10: Dropper with an incident wave in the CW, represented by $\Phi_{i}$ where $\Phi$ is defined in (14), generating 3 transmitted waves, one reflected wave and a force in the dropper.

The dropper is the only link between contact and messenger wires (see CW and MW in figure 1). It is non-linear when compressed over its initial tension. This is accounted for in FEM simulations but will be ignored here since waves generate low amplitudes. The dropper model is thus a spring/damper with two point masses representing claws linking wires to the dropper (visible in figure $2 \mathrm{~b}$ next to the sensor). This model is shown in figure 10 with an incident wave denoted $\Phi_{i}(k, \omega)$ propagating in the CW generating a reflected wave $\Phi_{r}$ and three transmitted waves. The problem, being linear, can be solved in the Fourier domain and all $\Phi(k, \omega)$ are the double Fourier transform, in space and time of their corresponding displacement $z(x, t)$,

$$
z(x, t)=\int_{-\infty}^{\infty} \int_{-\infty}^{\infty} \Phi(k, \omega) \mathrm{e}^{\mathrm{i} \omega(t \pm x / c)} d k d \omega
$$

where $c$ is the wave velocity in the cable and with \pm positive when the wave is propagating in the negative axis, namely $\Phi_{t-, \mathrm{MW}}$ and $\Phi_{r}$, and negative otherwise.

Equilibrium at claws $m_{\mathrm{CW}}$ and $m_{\mathrm{MW}}$ gives equations,

$$
\left\{\begin{array}{l}
\frac{T_{\mathrm{CW}}}{c_{\mathrm{CW}}}\left[\Phi_{i}-\Phi_{r}-\Phi_{t+, \mathrm{CW}}\right]-F_{\mathrm{drop}}=\mathrm{i} \omega m_{\mathrm{CW}} \Phi_{t+, \mathrm{CW}} \\
\frac{T_{\mathrm{MW}}}{c_{\mathrm{MW}}}\left[-\Phi_{t-, \mathrm{MW}}-\Phi_{t+, \mathrm{MW}}\right]+F_{\mathrm{drop}}=\mathrm{i} \omega m_{\mathrm{MW}} \Phi_{t+, \mathrm{MW}}
\end{array}\right.
$$

Continuity of displacement at $m_{\mathrm{CW}}$ and $m_{\mathrm{MW}}$ gives two more equations,

$$
\left\{\begin{array}{l}
\Phi_{i}+\Phi_{r}=\Phi_{t+, \mathrm{CW}} \\
\Phi_{t-, \mathrm{MW}}=\Phi_{t+, \mathrm{MW}}
\end{array}\right.
$$

and the impedance definition of the dropper gives

$$
F_{\text {drop }}=\left[\Phi_{m_{\mathrm{MW}}}-\Phi_{m_{\mathrm{CW}}}\right]\left(D+\frac{K}{\mathrm{i} \omega}\right)
$$

The system can be rewritten in matrix form as

$$
\left[\begin{array}{ccccc}
\frac{T_{\mathrm{CW}}}{c_{\mathrm{CW}}} & \frac{T_{\mathrm{CW}}}{c_{\mathrm{CW}}}+\mathrm{i} \omega m_{\mathrm{CW}} & 0 & 0 & 1 \\
0 & 0 & \frac{T_{\mathrm{MW}}}{c_{\mathrm{MW}}} & \frac{T_{\mathrm{MW}}}{c_{\mathrm{MW}}}+\mathrm{i} \omega m_{\mathrm{MW}} & -1 \\
-1 & 1 & 0 & 0 & 0 \\
0 & 0 & -1 & 1 & 0 \\
0 & D+\frac{K}{\mathrm{i} \omega} & 0 & -D-\frac{K}{\mathrm{i} \omega} & 1
\end{array}\right]\left\{\begin{array}{c}
\Phi_{r} \\
\Phi_{t+, \mathrm{CW}} \\
\Phi_{t-, \mathrm{MW}} \\
\Phi_{t+, \mathrm{MW}} \\
F_{\mathrm{drop}}
\end{array}\right\}=\left[\begin{array}{c}
\frac{T_{\mathrm{CW}}}{c_{\mathrm{CW}}} \\
0 \\
1 \\
0 \\
0
\end{array}\right]\left\{\Phi_{i}\right\}
$$


which is a an equation of the form $[A]\left\{\Phi_{i}(\omega)\right\}=[b]\left\{\Phi_{i}\right\}$ which can be inverted at each frequencies. The values $A^{-1} b$ correspond to a series of transfer functions

$$
\left\{\begin{array}{c}
\Phi_{r}(\omega) \\
\Phi_{t+, \mathrm{CW}}(\omega) \\
\Phi_{t-, \mathrm{MW}}(\omega) \\
\Phi_{t+, \mathrm{MW}}(\omega) \\
F_{\text {drop }}
\end{array}\right\}=\left[\begin{array}{c}
R_{\mathrm{drop}, \mathrm{CW}} \\
T_{\text {drop, CWtoCW }} \\
T_{\mathrm{drop}, \mathrm{CW} \text { toMW }} \\
T_{\mathrm{drop}, \mathrm{CWtoMW}} \\
Z_{\mathrm{drop}}
\end{array}\right]\left\{\Phi_{i}(\omega)\right\}
$$

where $T_{\mathrm{drop}, \mathrm{CWtoMW}}$ is the coefficient of transmission from the $\mathrm{CW}$ to the $\mathrm{MW}$ and $R_{\mathrm{drop}, \mathrm{CW}}$ is the coefficient of reflection of the wave in the $\mathrm{CW}$ reflected on the dropper.

Tests carried out are mass dropped under a dropper (see figure $2 \mathrm{~b}$ ). Incident waves are thus generated simultaneously in the CW and MW.

The same analytical work can be done for an incident wave coming from the MW leading to three other coefficients $R_{\mathrm{drop}, \mathrm{MW}}, T_{\mathrm{drop}, \mathrm{MWtoCW}}$ and $T_{\mathrm{drop}, \mathrm{MWtomW}}$. For a mass dropped at a dropper, two waves of the same amplitude are generated, $\Phi_{i, \mathrm{CW}}$ and $\Phi_{i, \mathrm{MW}}$ respectively in the contact and messenger wires. Figure 9 showed that this mass drop generates a step of velocity of amplitude $0.7 \mathrm{~m} / \mathrm{s}$.

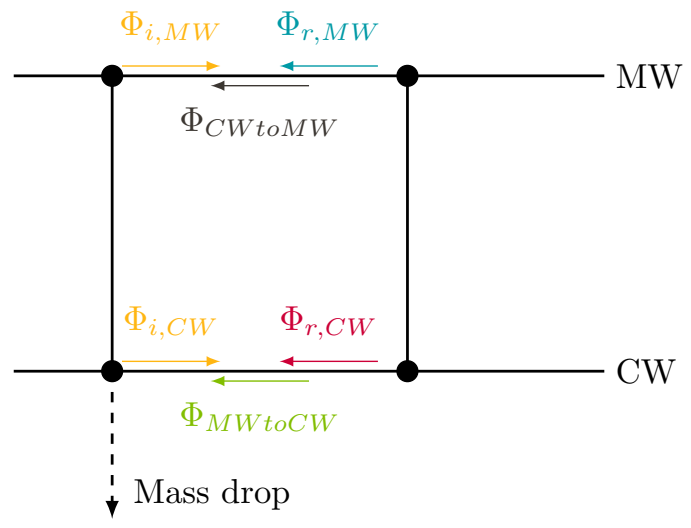

Figure 11: Incident $\Phi_{i}$, reflected $\Phi_{r}$ and transmitted $\Phi_{\mathrm{CWtoMW}}, \Phi_{\mathrm{CW}}$ tomW waves on a dropper after a mass drop at the dropper on the left.

The waves reflected and transmitted represented in figure 11, that reach back the initial dropper where the mass was dropped can be computed in the time domain by inverse Fourier transform of (14) and with the reflection/transmission coefficients determined in (19) respectively for $\Phi_{\mathrm{CWtoMW}}$ and $\Phi_{r, \mathrm{CW}}$. The two last, $\Phi_{\mathrm{MWtoCW}}$ and $\Phi_{r, \mathrm{MW}}$ are determined in a similar fashion, permuting contact and messenger wires. Figure 12 shows the vertical velocity of these waves at the observed dropper, separately (12a) and summed (12b). 


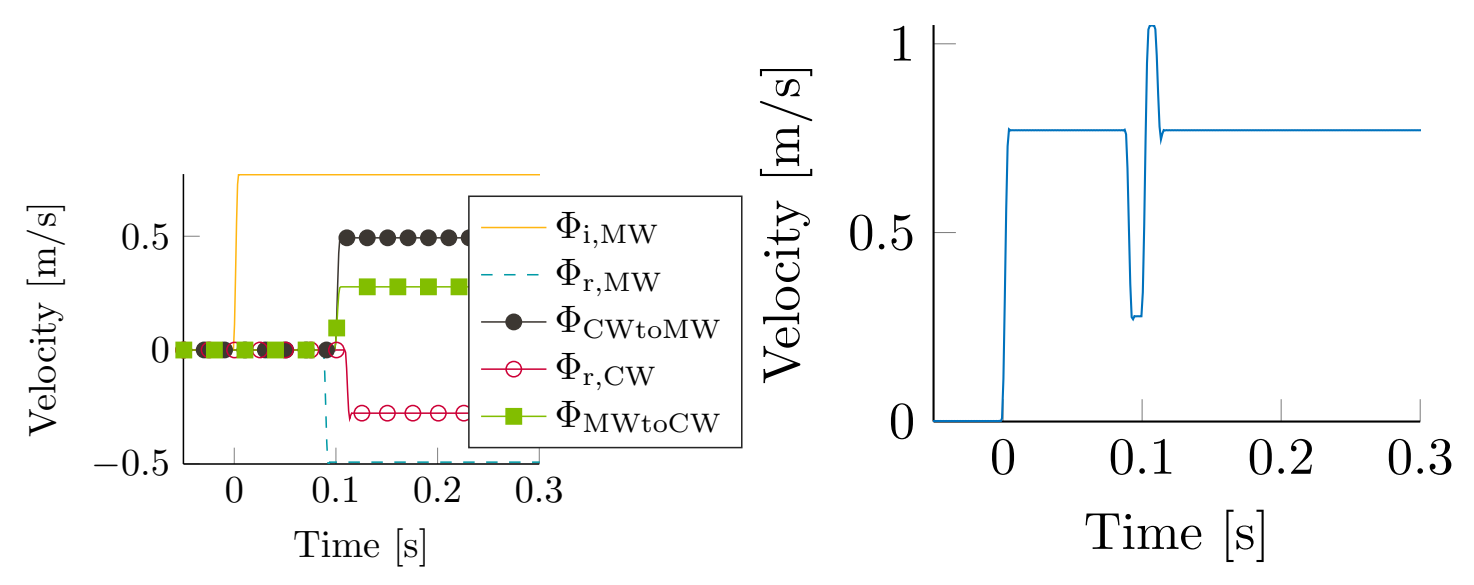

(a)

(b)

Figure 12: Wave velocities. (a) separated; (b)cumulated.

The analytical solution of the 40.6kg mass drop at position 1 in the configuration of figure 13a was shown in figure 12. The simulation was led in the same configuration where the next dropper was removed to avoid wave reflection on the right side, which makes results comparable to the analytic model. As shown in figure $13 \mathrm{~b}$, the main effect is a single oscillation near $0.1 \mathrm{~s}$ visible in the analytic string model. In the FEM result, this oscillation is distorted by the presence of higher frequency oscillations due to dispersion. In the test further distortion is induced by reflections on the next dropper, but the results are very similar.
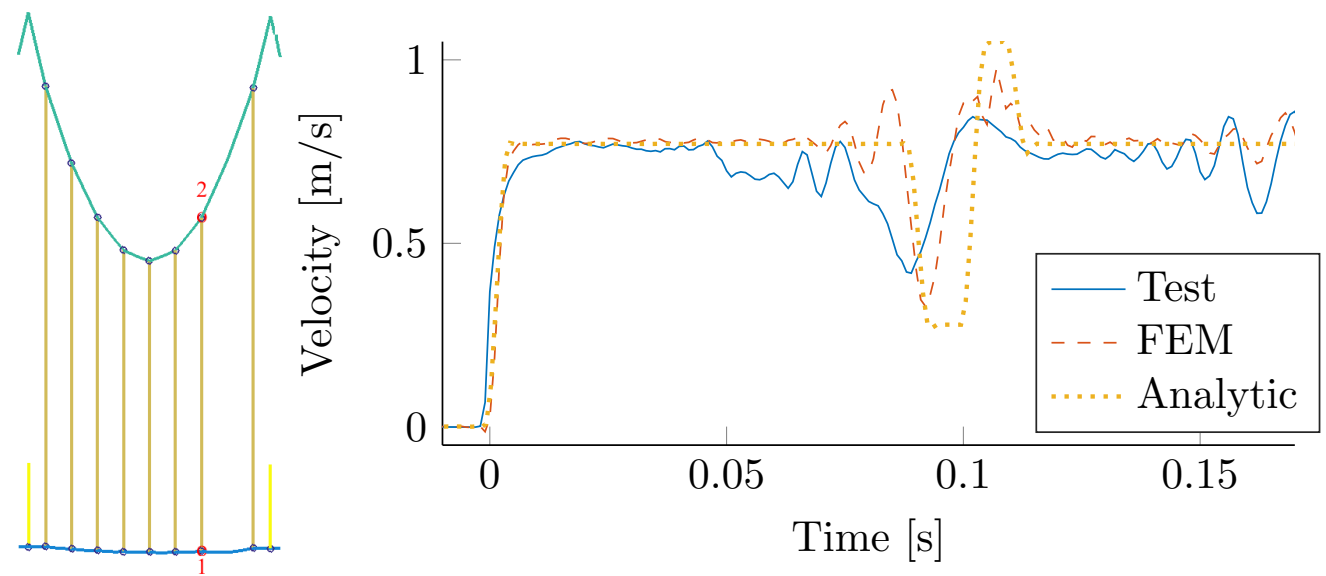

(a)

(b)

Figure 13: Dropper reflections. (a) Simulation configuration; (b) Simulation, measurements and analytical vertical velocity of the $\mathrm{CW}$ at mass drop.

The main conclusion at this point is that a compensation of velocity happens at droppers. The slight delay, if wave velocities differ in $\mathrm{CW}$ and $\mathrm{MW}$, only induces a short time variation.

\subsection{Wave behaviour around a mast}

The passage of the pantograph at the position of the mast is known to be the source of current collection issues with frequent arcing. At this position, the MW is linked to the mast and the $\mathrm{CW}$ is connected to the 
steady arm (see figure 2a).

In simulations, the MW is linked to the mast by a ball joint link, authorizing rotation in the transversal and vertical axis of the MW. Vertical displacement is thus not possible and the incident waves reaching this link are fully reflected using a string model and nearly fully in the beam case.

The steady arm shown in figure 2a is an elbow arm used in traction to apply a stagger to the CW. The steady arm is not exactly horizontal and the part attached to the bracket is around $20 \mathrm{~cm}$ higher than the $\mathrm{CW}$. The arm is however articulated which gives a very low stiffness in the radial direction and induces a light coupling between vertical and horizontal directions. Reflections on steady arm are thus neglected in the analytical model.

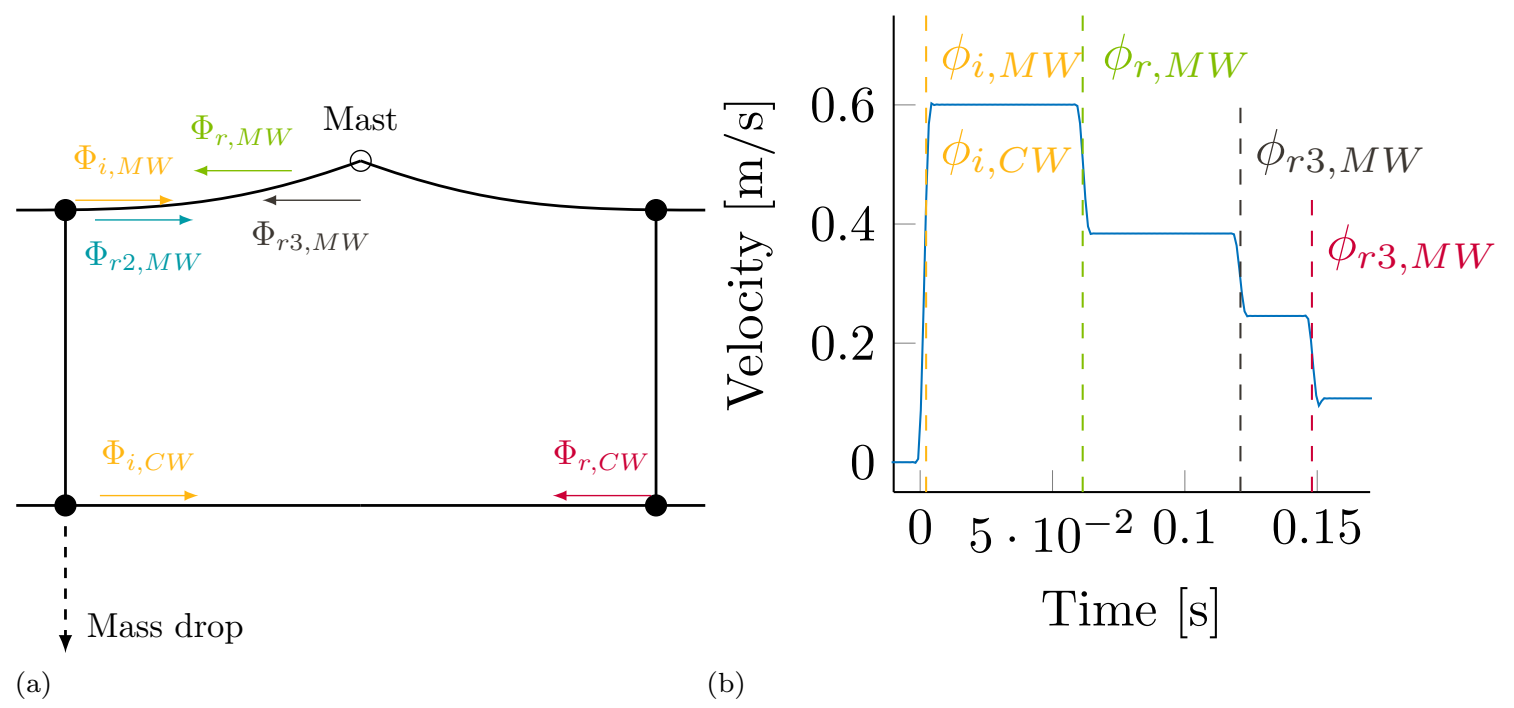

Figure 14: (a) Waves propagating in the catenary around mast after a mass drop under a dropper; (b) Analytical vertical velocity of the CW at the mass drop with flying times of reflections in vertical dashed lines.

The wave in the CW that has not been reflected by the SA is reflected later on the first dropper of the following span. This time, as the mast blocked MW transmission, velocity compensation does not occur and a change in level is expected.

For a mass dropped at the last dropper of a span, the wave $\Phi_{i, \mathrm{MW}}$ is fully reflected in the opposite side as $\Phi_{r, \mathrm{MW}}$. This new wave is again reflected on the dropper to generate $\Phi_{r 2, \mathrm{MW}}$ and on the mast to finally generate $\Phi_{r 3, \mathrm{MW}}$, which reaches the dropper where the mass was dropped before $\Phi_{r, \mathrm{CW}}$. The vertical velocity at the position of the mass drop is computed analytically and displayed in figure 14 .

Around mast, the successive total reflections on the mast in the MW and the reflections against the first dropper of the following span in the $\mathrm{CW}$ generate several level changes in vertical velocity observed where the mass is dropped. The corresponding simulation was led on the catenary shown in figure 15a with a mass of $40.6 \mathrm{~kg}$ dropped at position 1 . In figure $15 \mathrm{~b}$, analytical and simulation results are compared to a measurement in the configuration of figure $2 \mathrm{c}$, the mass being dropped at 1 (blue) and the observation at 4 (red). Simulation and analytical results are very close. The difference around $0.065 \mathrm{~s}$ is mainly due to wave reflections on the steady arm, which was not taken into account in analytical model. After $0.2 \mathrm{~s}$, waves generated on the left side and reflected on droppers start to disrupt simulation results. For the measurement, the observed change of velocity level is the same since wave reflections on droppers only induce oscillations around these levels. 


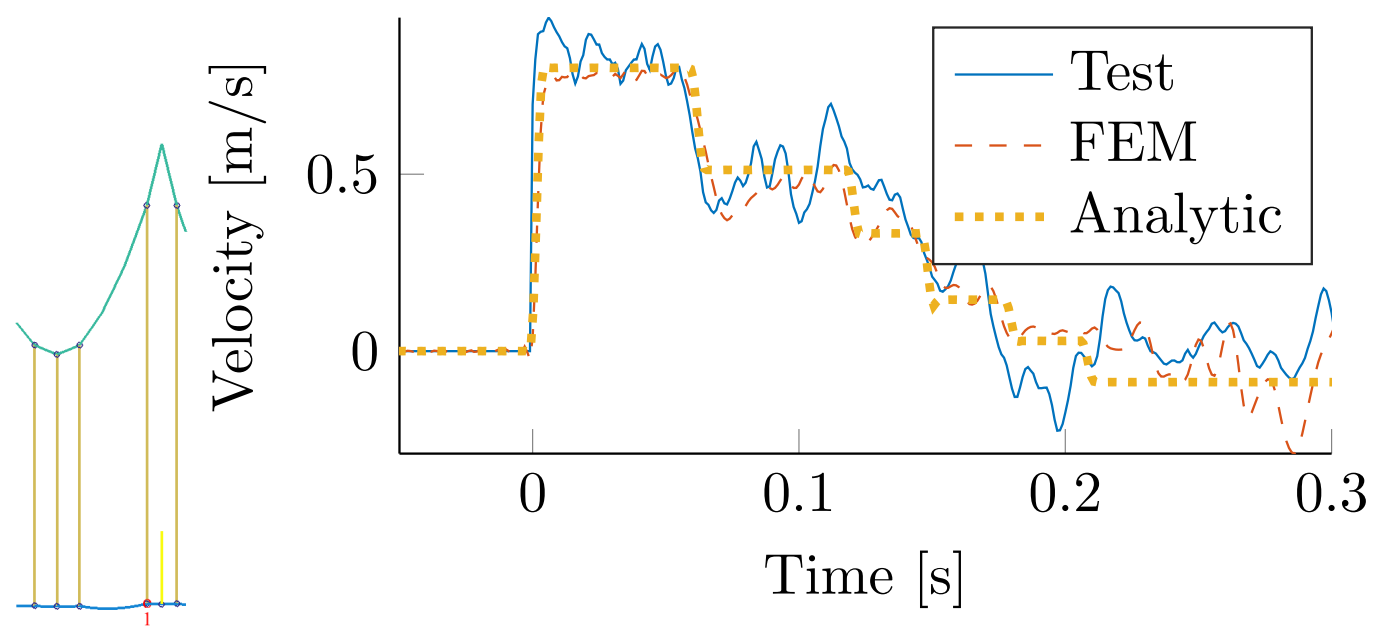

(a)

(b)

Figure 15: Mast reflections. (a) Simulation configuration; (b) Simulation, measurements and analytical vertical velocity of the $\mathrm{CW}$ at mass drop.

The amplitude of all steps observed in this signal depend on the coefficients of reflection/transmission computed in (19). At frequency of $0 \mathrm{~Hz}$ they can be written as

$$
\begin{aligned}
T_{\mathrm{drop}, \mathrm{CWtoCW}}(\omega=0)= & T_{\mathrm{drop}, \mathrm{CWtoMW}}(0)=-R_{\mathrm{drop}, \mathrm{MW}}(0) \\
= & \frac{\frac{T_{\mathrm{CW}}}{c_{\mathrm{CW}}}}{\frac{T_{\mathrm{CW}}}{c_{\mathrm{CW}}}+\frac{T_{\mathrm{MW}}}{c_{\mathrm{MW}}}} \\
T_{\mathrm{drop}, \mathrm{MWtoMW}}(\omega=0)= & T_{\mathrm{drop}, \mathrm{MWtoCW}}(0)=-R_{\mathrm{drop}, \mathrm{CW}}(0) \\
= & \frac{\frac{T_{\mathrm{MW}}}{c_{\mathrm{MW}}}}{\frac{T_{\mathrm{MW}}}{c_{\mathrm{MW}}}+\frac{T_{\mathrm{CW}}}{c_{\mathrm{CW}}}}
\end{aligned}
$$

with $T_{\mathrm{CW}}$ and $T_{\mathrm{MW}}$ the mechanical tensions.

For the catenary on which tests were made, a wave generated in the CW is lightly reflected and mainly keeps propagating while generating two waves in the MW of amplitude equal to the transmitted wave. Once in the MW, the wave is mainly reflected at dropper and less transmitted to the three other parts. A wave in the MW is thus more likely to be trapped between droppers than transferred to the CW.

But in usual catenary designs, $\frac{T_{\mathrm{CW}}}{c_{\mathrm{CW}}}$ is close to $\frac{T_{\mathrm{MW}}}{c_{\mathrm{MW}}}$, which leads to coefficients close to 0.5 and an equipartition of waves in contact and messenger wires. For the rest of the study, the transmission coefficient at zero frequency

$$
a=\frac{\frac{T_{\mathrm{CW}}}{c_{\mathrm{CW}}}}{\frac{T_{\mathrm{CW}}}{c_{\mathrm{CW}}}+\frac{T_{\mathrm{MW}}}{c_{\mathrm{MW}}}}
$$

is introduced to quantify wave repartition. The coefficients $\frac{T_{\mathrm{CW}}}{c_{\mathrm{CW}}}$ is the dynamic impedance of the $\mathrm{CW}$ and 
can be written as a function of $T_{\mathrm{CW}}$ and $\mu_{\mathrm{CW}}$, the linear mass of the cable. The wave velocity and impedance

$$
\left\{\begin{array}{l}
c_{\mathrm{MW}}=\sqrt{\frac{T_{\mathrm{MW}}}{\mu_{\mathrm{MW}}}} \\
\frac{T_{\mathrm{MW}}}{c_{\mathrm{MW}}}=\sqrt{\mu_{\mathrm{MW}} T_{\mathrm{MW}}}
\end{array}\right.
$$

is thus more representative to quantify dynamic impact of waves than $T_{\mathrm{CW}}$ and $\mu_{\mathrm{CW}}$.

\section{Time of flight, fixed and moving frames}

Pantograph passage on stiff points (droppers, SA) generates waves, which when reflected will generate response fluctuations. Since such elements are repeated, the response will be expected to have periods (in time/space) or harmonics (in frequency/wavenumber) combining spatial periodicity, train velocity and wave velocity. The periods are estimating a time of flight analysis. Section 5.1 deals with responses in the fixed frame of the catenary and section 5.2 with those in the moving frame of the pantograph. Section 5.3 finally details other coincidences of interest.

\subsection{Fixed frame}

Section 3.2 illustrated that the contact force due to the catenary geometry is mostly spatially periodic with a fundamental spatial frequency corresponding to the span length $d_{\text {span }}$. At each peak of force, a wave is generated. Supposing this wave is, for the sake of simplicity, generated at the steady arm $S A_{1}$ at $t=0$, if the vertical velocity of the catenary is observed later at $x_{\mathrm{obs}}$ as shown on figure 16 , the wave reaches the observation point with the delay

$$
t_{1}=\frac{d_{1}}{c_{\phi}}
$$

When the pantograph reaches the next steady arm $S A_{2}$ at $t=\frac{d_{\text {span }}}{v_{\text {panto }}}$, it generates another wave which reaches $x_{\mathrm{obs}}$ placed at a distance $d_{1}-d_{\text {span }}$ to the dropper after a delay

$$
t_{2}-\frac{d_{\text {span }}}{v_{\text {panto }}}=\frac{d_{1}-d_{\text {span }}}{c_{\phi}} .
$$

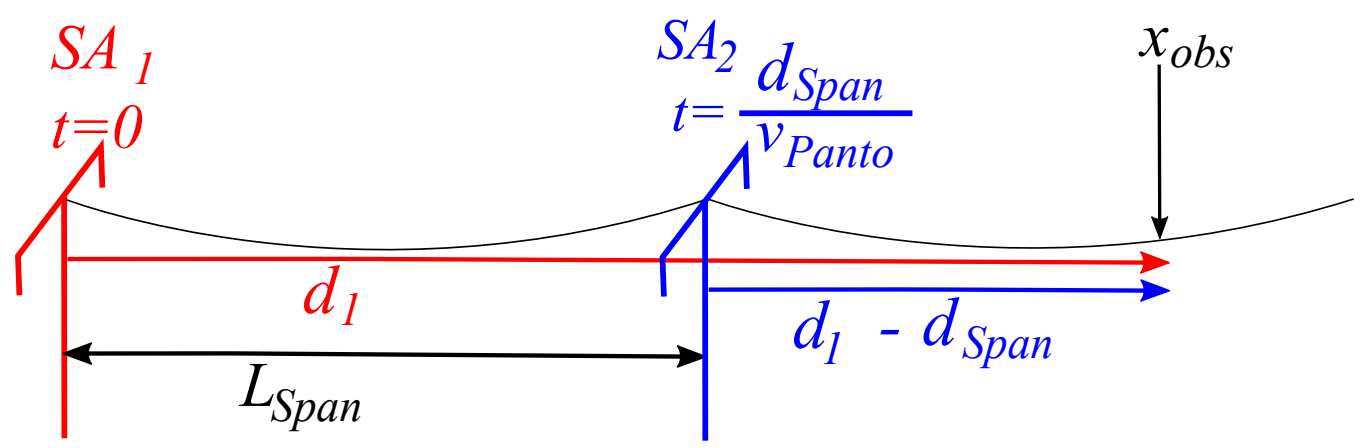

Figure 16: Wave propagation illustration, view from catenary.

The characteristic period of wave propagated by successive passage on points regularly distant of $d_{\text {span }}$ is thus

$$
t_{2}-t_{1}=d_{\text {span }}\left(\frac{1}{v_{\text {panto }}} \pm \frac{1}{c_{\mathrm{CW}}}\right)=\frac{d_{\text {span }}}{v_{\text {panto }}}(1 \pm \beta)
$$

with "-" when $x_{\text {obs }}$ is after the pantograph and "+" when $x_{\text {obs }}$ is behind.

To illustrate these characteristic times, figure 17b shows the uplift induced by the waves generated by the pantograph passing successive steady arms. Figure 17a shows a map of the velocity of uplift for each 
position of the catenary against time. The solid black line indicates the pantograph position, the red lines highlight the waves generated by the pantograph when it passes under a steady arm and the black dotted line indicates the position of the point observed on the right figure.

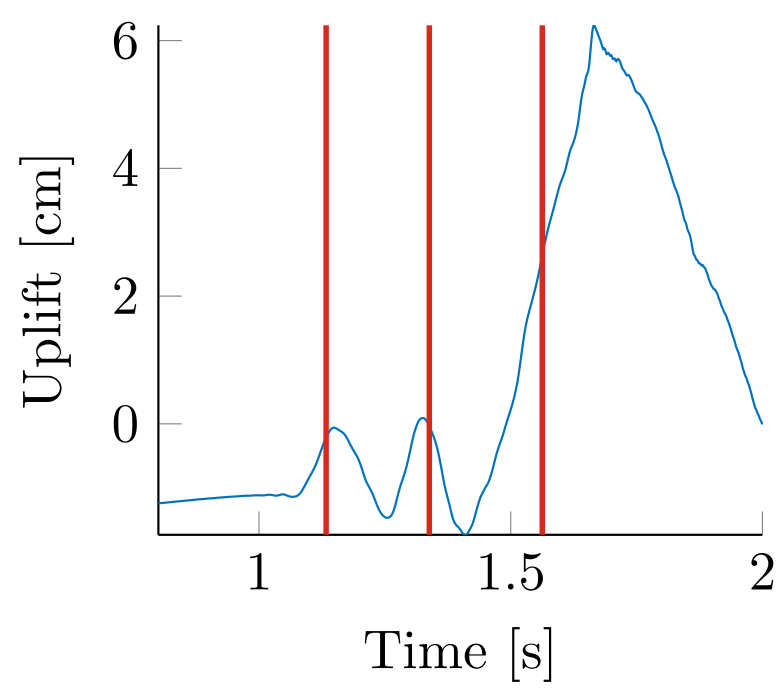

(a)

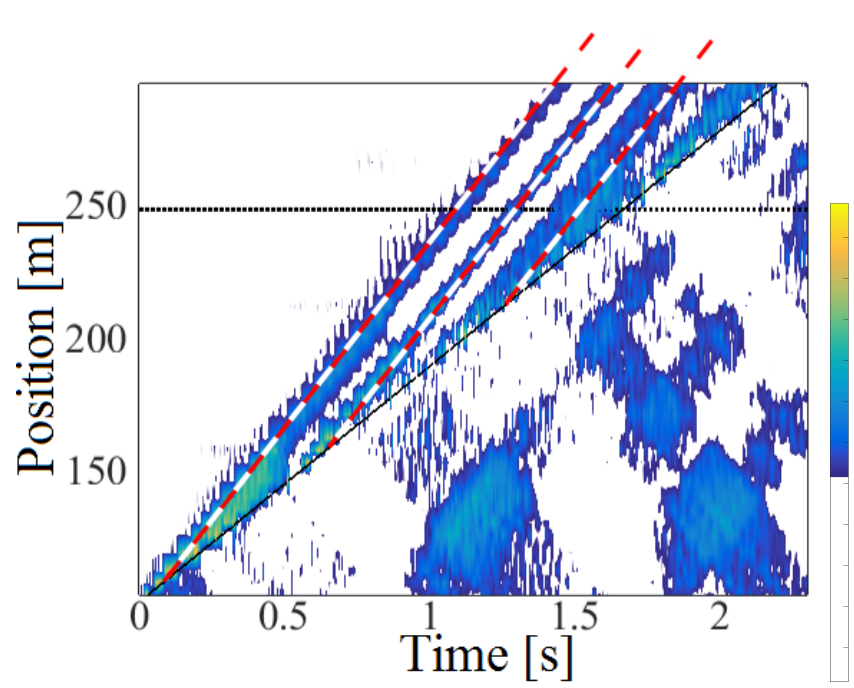

(b)

Figure 17: (a) Uplift of the catenary at a position $x=247.5 m$; (b) Map of velocity of uplift along the catenary against time.

As the generated signal is periodic, its Fourier transform will be composed of all harmonics of the base frequency $1 /\left(t_{2}-t_{1}\right)$. Spectra at fixed locations are thus expected to high levels at frequencies

$$
f_{\text {wave }, \text { cat }, \pm}=\frac{n \cdot v_{\text {panto }}}{d_{\text {span }}(1 \pm \beta)} \quad \forall n \in \mathbb{N}^{*}
$$

Introducing $k_{\text {span }}=1 / d_{\text {span }}$ first defined in (3) leads, in the spatial frequency domain, to

$$
k_{\text {wave,cat }, \pm}=\frac{n}{1 \pm \beta} k_{\text {span }} \quad \forall n \in \mathbb{N}^{*}
$$

Figure 18 shows these frequencies on map of amplitudes of vertical wire velocities as a function of frequency and train velocity. Analytical definitions of the harmonics, shown as black curves, clearly coincide with areas of higher amplitude in the spectra. The network of harmonics is particularly clear when it coincides with modal groups of the catenary which will be further detailed in section 6 . 


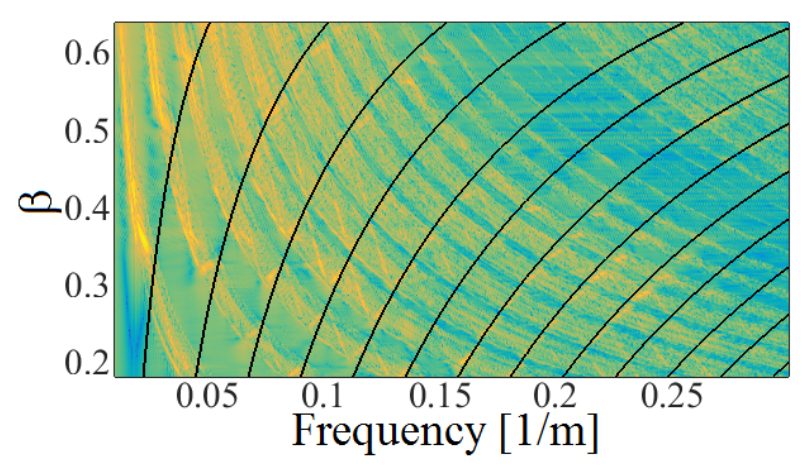

(a)

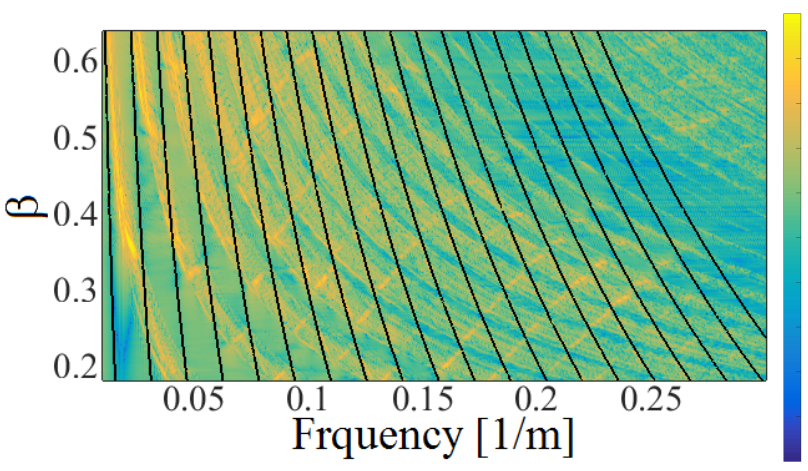

(b)

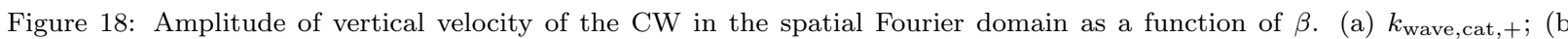
$k_{\text {wave,cat, }- \text {. }}$

\subsection{Moving Frame}

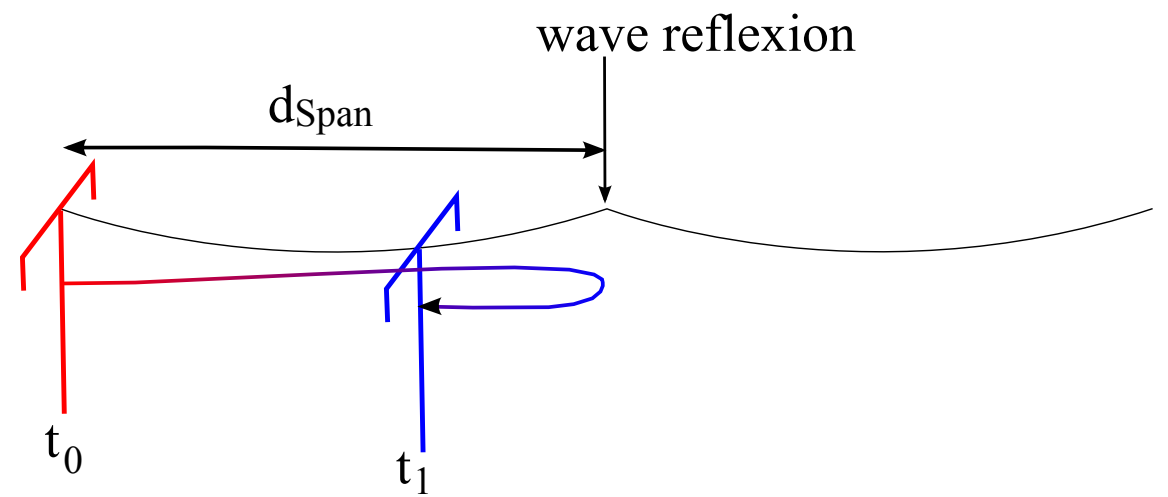

Figure 19: Wave propagation illustration, view from pantograph.

From the pantograph point of view, the characteristic times are described in figure 19 where the pantograph moves under a periodic profile of spatial period $d_{s}$. At $t_{0}$, the pantograph passes under a change (steady arm or dropper) and generates waves forward and backward which reflect on the next or previous changes and reach the pantograph at $t_{1}$ after the pantograph has moved by a distance $d_{1}$ which can be written as

$$
t_{1}-t_{0}=\frac{2 d_{s} \pm d_{1}}{c_{\mathrm{CW}}}=\frac{d_{1}}{v_{\text {panto }}}
$$

with " -" when propagating forward and " +" when propagating backward.

From this equation, the distance $d_{1}$ can be computed as

$$
d_{1}=2 d_{s} \frac{\beta}{1 \pm \beta}
$$

and a characteristic spatial frequency by

$$
k_{\text {wave,panto, } \pm}=\frac{1 \pm \beta}{2 \beta} k_{s}
$$

with $k_{s}=1 / d_{s}$. 
Figure 20 shows the contact force simulated along one span and as a function of $\beta$. The black lines indicate mast positions, grey lines indicate dropper positions and red curves indicate the position where the pantograph is supposed to meet the wave generated at passage under the previous dropper and reflected on the next dropper. For higher $\beta$, the point where the pantograph meets the reflection gets closer to the next dropper.

The dropper scale is the smallest in the spatial domain and thus the one observed here. The span scale, which is the smallest in frequency domain, is hard to distinguish in spatial domain.

On this figure, a change of behaviour can be observed around $\beta=0.33$. This corresponds to the pantograph speed $v_{\text {panto }}$ after which the backward wave meets the pantograph in a different span. At that particular velocity, the backward wave reflection meets the pantograph exactly after this span.

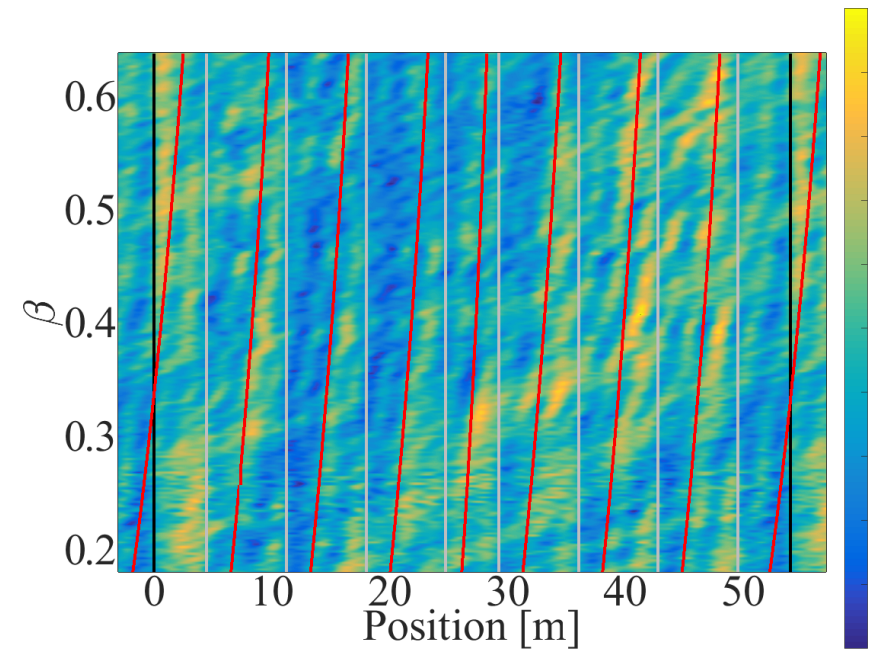

Figure 20: Contact force along one span as a function of $\beta$.

Visualizing the relevance of characteristic flight times does not imply knowledge of where maxima occur. The contact force is highly dependent on the catenary geometry as explained in section 3 and figure 20 shows that the maxima of contact force are not placed under droppers.

The approximation of $F_{c}$ by $F_{\text {geom }}$ displayed in figures 8 and 7 showed that the amplitudes of Fourier transforms are easier to compare than spatial signals. Characteristic times are thus converted to characteristic spatial frequencies and the distance $d_{\text {span }}$ is replaced by all the spatial frequencies of peaks of $V_{\mathrm{CW}}$ shown in figure $5 \mathrm{~b}$. These frequencies are multiples of the span spatial frequency, $k_{\mathrm{span}}=1 / d_{\text {span }}$ and wave frequencies can be defined as

$$
k_{\text {wave }, \text { panto }, \pm}=\frac{1 \pm \beta}{\beta} \frac{n}{2} k_{\text {span }} \quad \forall n \in \mathbb{N}^{*}
$$

Figure 21 shows an amplitude map of vertical velocity of the CW in the spatial Fourier domain as a function of $\beta$ and the curves corresponding to $f_{\text {wave,panto, }+}$ on the left and $f_{\text {wave,panto,- }}$ on the right. While such lines seem to appear, other effects dominate the response and make the analysis difficult. 


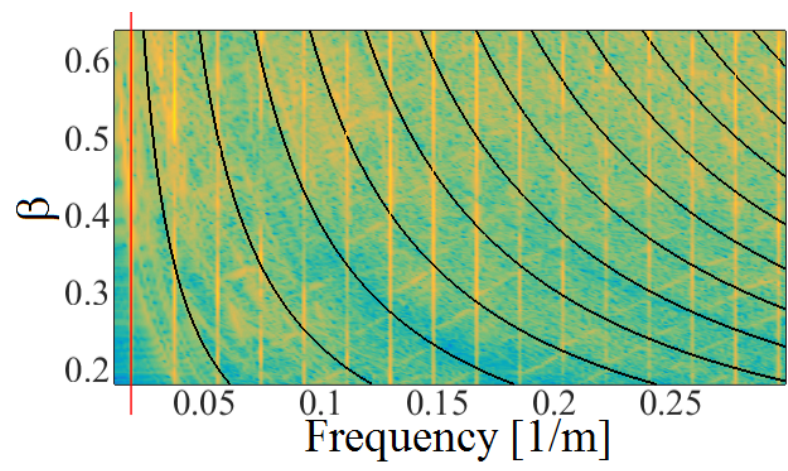

(a)

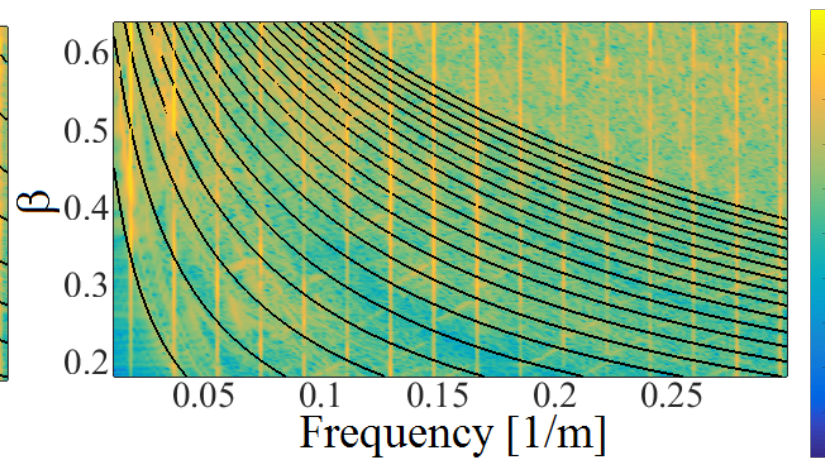

(b)

Figure 21: Amplitude of the contact force $F_{c}$ in the spatial Fourier domain as a function of $\beta$. (a) $k_{\text {wave,panto,+; }}$ $k_{\text {wave,panto,- }}$.

\subsection{Other coincidences}

The amplitude of the first vertical line of the contact force amplitude spectrum shown in figure 21 as a red line, increases with $\beta$ in the same way as $F_{\text {geom }}$ determined in section 3 but with small fluctuations. These fluctuations are clearer if the phase of this fundamental frequency is compared to that of $F_{\text {geom }}$ as shown in figure 22 .

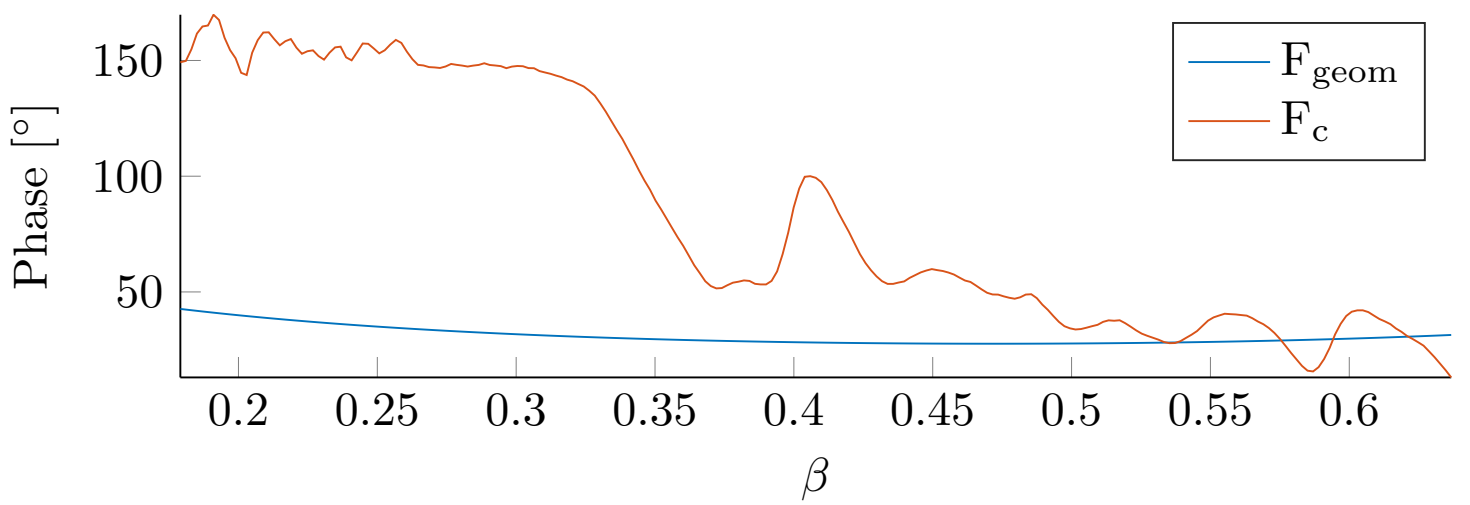

Figure 22: Phase of contact force of the fundamental spatial frequency $k_{\text {span }}$ as a function of $\beta$.

The notable phase gap for $\beta<0.33$ corresponds to low velocity domain where waves reflected successively on two spans meet the pantograph in the same span as shown with figure 20 . It is also the velocity under which reflections of waves generated backward the pantograph reach the pantograph in the same span.

The peak at $\beta=0.4$ correspond to the particular pantograph velocity $v_{\text {panto }}$ at which the span passage temporal frequency $f_{\text {span }}=k_{\text {span }} \cdot v_{\text {panto }}$ coincides with the first group of vertical catenary modes around $1 \mathrm{~Hz}$ (see section 6). In our case, $k_{\mathrm{span}}=1 / 54 \mathrm{~m}^{-1}$ and the span passage frequency is equal to $1 \mathrm{~Hz}$ for $v_{\text {panto }}=54 \mathrm{~m} / \mathrm{s}$, which gives, for $c_{\mathrm{CW}}=c_{\mathrm{MW}}=138 \mathrm{~m} / \mathrm{s}, \beta=0.4$.

The importance of this specific coincidence appeared in a study where the current collection was shown to be better for $\beta=0.4$. The prior knowledge of where relevant spectral lines are expected can thus be used to find appropriate objective functions (here the phase of the first spectral line). In the present case, the contact force amplitude spectrum is lower for higher phases and this corresponds to a case where the pantograph meets the reflected waves under droppers or steady arms. 


\section{Catenary modes}

Catenary modes are closely linked with waves but for large wavelengths at low frequencies. Section 6.1 details the existence of mode groups and their structure. The impact of modes on multi pantograph configurations is seen in section 6.2. Variations of mechanical properties of wires are then introduced in section 6.3 to define the proper characteristic wave velocity in the catenary.

\subsection{Modes frequencies}

The aim of this section is to identify frequencies linked with catenary modes. To analyse these modes, simulations were led for a pantograph running over $3000 \mathrm{~m}$ even if the catenary is only $1100 \mathrm{~m}$ long so that the frequency resolution is increased in the Fourier domain. The pantograph impact is thus reduced and the free response of the catenary is more pronounced.

On the map displayed in figure 23, the first obvious lines observed are those which do not change with pantograph velocity $v_{\text {panto }}$ and are thus vertical in the temporal Fourier domain. These lines correspond to the modal frequencies of the catenary and the vertical black lines indicate these frequencies for the first two groups of modes, under $3 \mathrm{~Hz}$.

To understand the origin of mode groups, it is useful to see that the spans are only coupled by the steady arm, since the MW is blocked at the mast. For a blocked steady arm, each span would have identical modes and the section would have modes with multiplicity equal to the number of spans. As the steady arm has strong lateral but low vertical stiffness, some level of inter-span coupling exists. As a result the identical span modes actually couple and form a group with a certain spread in frequency. Thus there are as many modes in the first group under $1.5 \mathrm{~Hz}$, as spans in the catenary section.

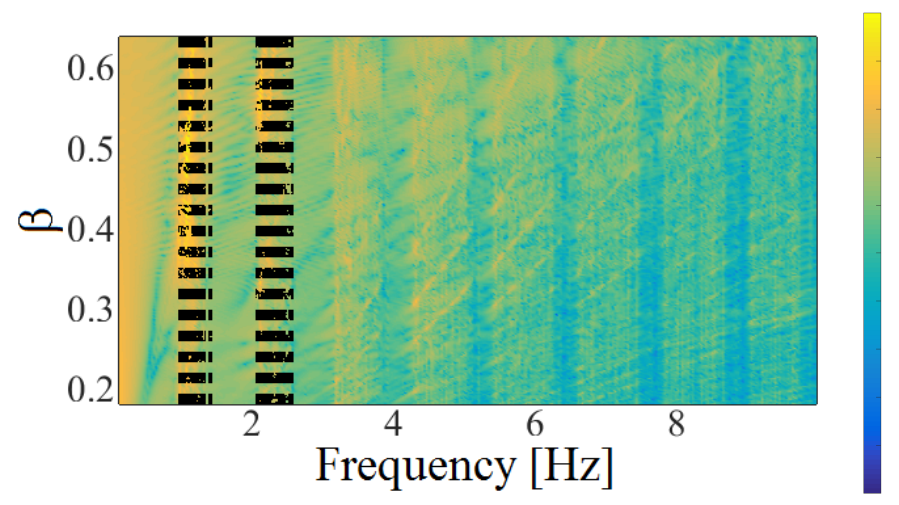

Figure 23: Amplitude of vertical velocity of the CW in the temporal Fourier domain as a function of $\beta$ with catenary modes indicated as vertical lines.

Figure 24 shows a mode of the first group where the first span bending mode (highest amplitude at mid-span and low level on edges) is observed in every span, but with a different amplitude for each thus illustrating coupling.

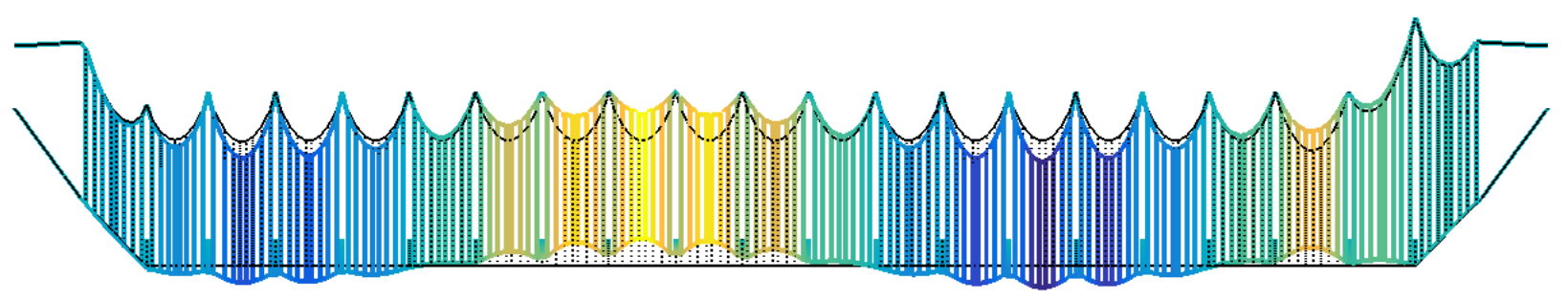

Figure 24: Mode 3 of the catenary section at $1.054 \mathrm{~Hz}$. 
At higher frequencies, coupling increases so that the group spread is larger and one tends to have overlap of different groups. Introducing non-periodic spans also leads to wider mode bands.

\subsection{Multi-pantograph configurations}

High speed trains are often composed of two train sets and are thus equipped with two pantographs collecting current simultaneously. The current collection quality of the second pantograph strongly depends on the dynamic state of the catenary after the passage of the first pantograph. It is thus highly sensitive to the distance between pantographs, $d_{\text {panto }}$. The usual distance $d_{\text {panto }}$ is of $200 \mathrm{~m}$, the length of a train set.

Figure 25 shows the current collection quality criterion $\sigma / F_{m}$ defined in (1) as a function of distance $d_{\text {panto }}$ and normalized velocity $\beta, \sigma$ being the standard deviation of the $70 \mathrm{~Hz}$-filtered contact force and $F_{m}$ its mean. This criterion shows oscillations with $d_{\text {panto }}$ and the period of these oscillations increases non-regularly with $\beta$.

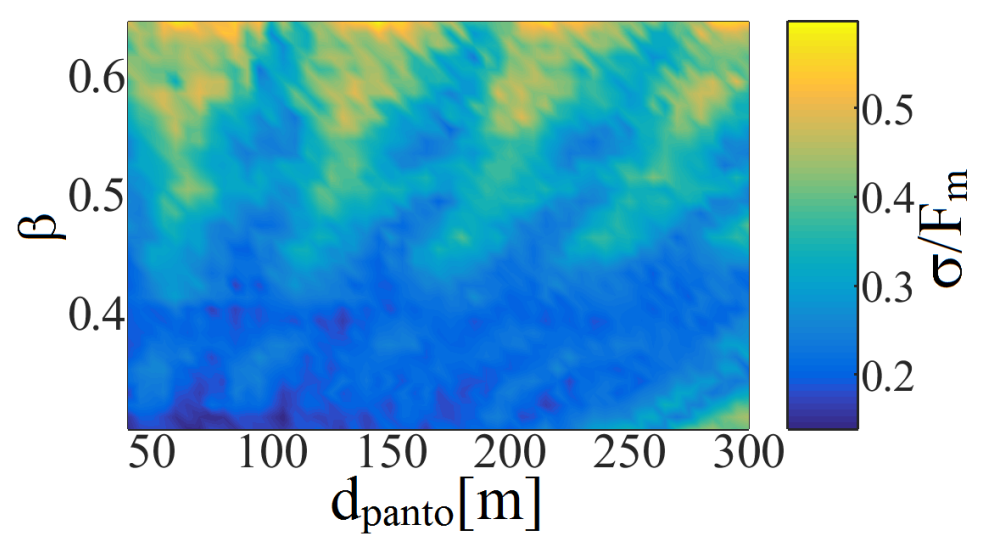

Figure 25: $\sigma / F_{m}$ of the trailing pantograph as a function of $\beta$ and $d_{\text {panto }}$

The variation with $\beta$ of the periodic oscillations along $d_{\text {panto }}$ shows that the phenomenon is not due to geometry, otherwise it would not depend on $\beta$. Catenary vibration modes are thus assumed to be the cause.

Denoting the modal frequencies $f_{\text {modes }}$, the spatial period $d_{\text {modes }}$ can be defined as

$$
d_{\text {modes }}=\frac{v_{\text {panto }}}{f_{\text {modes }}} \text {. }
$$

Figure 26 shows the evolution of multiples of $d_{\text {modes }}$ for the first group of modes as a function of $\beta$ compared to the map of $\sigma / F_{m}$ normalized for each $\beta$ in order to highlight low velocity behaviour. Vertical displacement of the contact wire is higher for the first group of modes. Furthermore, all maxima of $\sigma / F_{m}$ are approximately placed inside the bands defined by $d_{\text {modes }}$ multiples even if noticeable variations are observed inside these bands. 


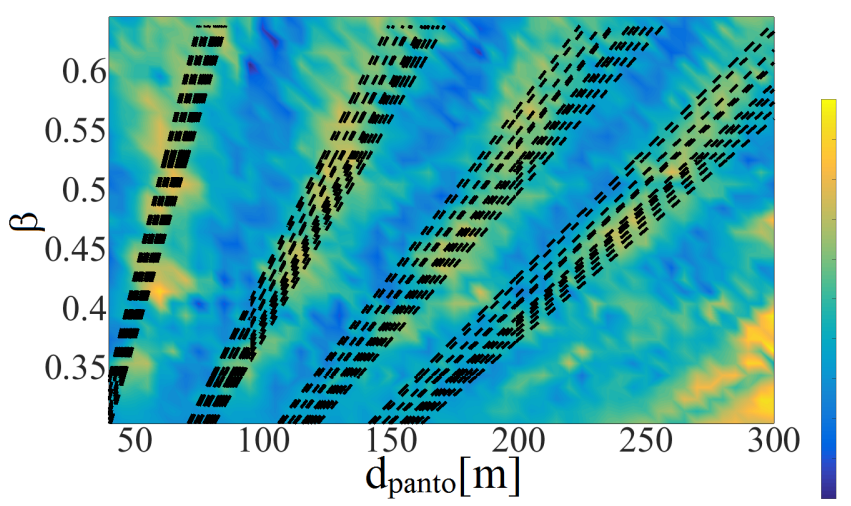

Figure 26: $\sigma / F_{m}$ of the trailing pantograph as a function of $\beta$ and $d_{\text {panto. }}$.

Figure 27 shows the amplitude of the catenary vertical displacement spectrum on the left and a zoom on frequencies around $1 \mathrm{~Hz}$. The first peak slightly changes with $\beta$. For $\beta$ increasing between 0.4 and 0.6 , the frequency increases and thus distance $d_{\text {modes }}$ decreases, which is consistent with observations in Figure 26. Evolution of the frequency as a function of $\beta$ is due to the coupling with the pantograph, changing dynamic properties of the assembled system.

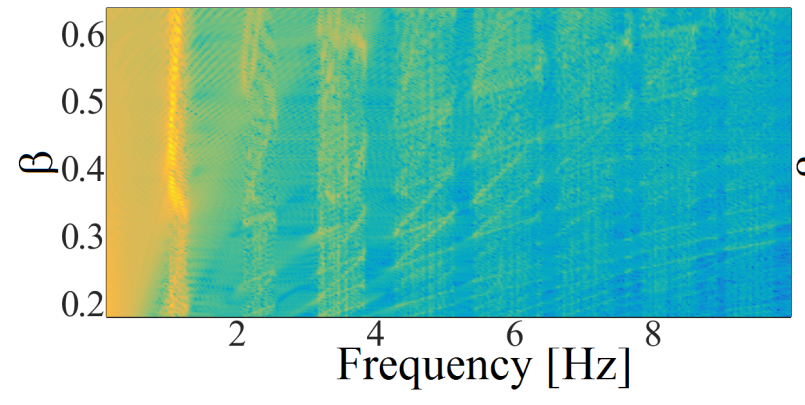

(a)

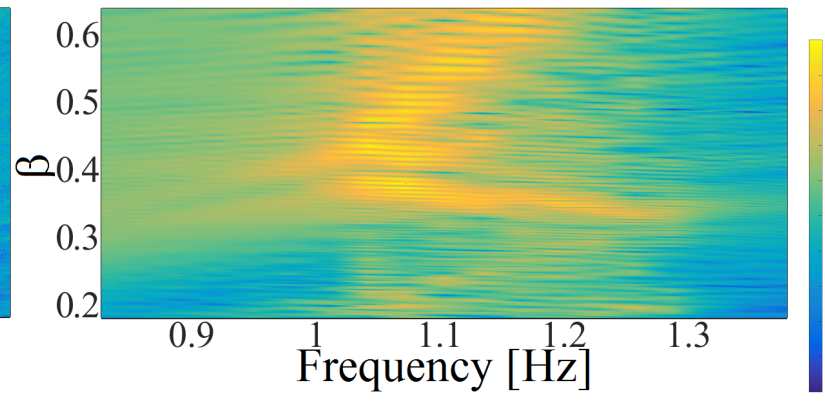

(b)

Figure 27: Amplitude of the spectrum of vertical displacement of one CW point (catenary uplift) as a function of $\beta$. (a) Large frequency range; (b) zoom on $1 \mathrm{~Hz}$.

Figure 28a shows the vertical velocity of a point fixed on catenary as a function of $\beta$ and of the distance between the pantograph and the observation point, for simulations led with one pantograph only. On Figure $28 \mathrm{~b}$, the map observed previously is shown again as a comparison. It is clear that the current collection quality of the trailing pantograph is closely linked with the catenary vertical velocity seen by the trailing pantograph. When this velocity is at its maximum, the current collection is better. 


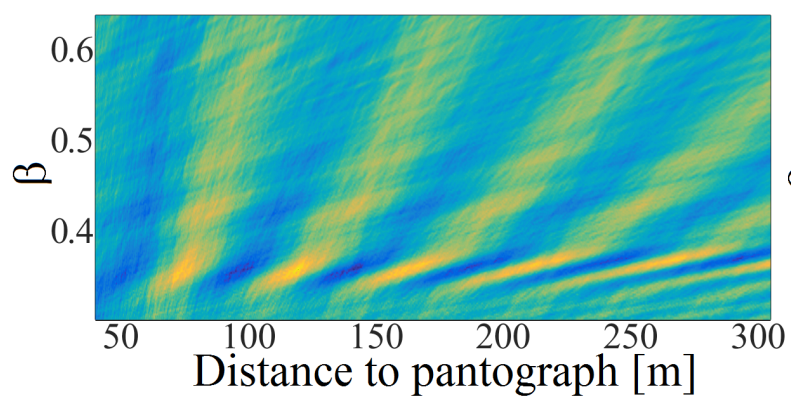

(a)

Figure 28: (a) Vertical velocity of the $\mathrm{CW}$ as a function of $\beta$ and of the distance between the pantograph and the observation point; (b) $\sigma / F_{m}$ of the trailing pantograph as a function of $\beta$ and $d_{\text {panto. }}$.

The distance $d_{\text {panto }}$ can be optimized for a given catenary and a given speed. A periodic catenary induces a tighter band of first frequency modes and thus amplifies the phenomenon. On the contrary, to lower variability with speed and $d_{\text {panto }}$ of current collection quality of the trailing pantograph, spreading mode bands by increasing coupling or braking periodicity at span scale seems effective.

\subsection{Variations with mechanical properties of wires}

Section 4.3 illustrated that the influence of the MW properties can be dissociated into two relevant parameters: the dynamic impedance $\sqrt{\mu_{\mathrm{MW}} T_{\mathrm{MW}}}$ and the wave velocity $c_{\mathrm{MW}}$. This section considers variations of $c_{\mathrm{MW}}$ while keeping constant the dynamic impedance by changing the MW section. Figure 29 shows the catenary modes computed on a full catenary (left) and on a $54 \mathrm{~m}$ span (right).

Modes that quickly change with $c_{\mathrm{MW}}$ correspond to horizontal modes of the MW illustrated in Figure 30a. The horizontal stiffness of droppers being close to zero, these modes have very little coupling with the $\mathrm{CW}$ leading to the high sensitivity to $c_{\mathrm{MW}}$. The frequency spread of the band is very small showing that these modes are nearly independent between spans. Modes that stay stable are the horizontal modes of the CW. Once again, the bandwidth is small showing that the horizontal stiffness induced by steady arms makes spans independent for these modes.

The last type of mode groups corresponds to vertical modes coupling CW and MW. The frequency spread indicates that spans are coupled and the intermediate sensitivity to $c_{\mathrm{MW}}$ indicates that the energy is balanced between the CW and MW. These vertical modes are those excited by pantograph motion and visible in Figure 23. Important modes are thus dependent on velocities in both the CW and MW. The value currently thought to be relevant is the mean $\left(c_{\mathrm{CW}}+c_{\mathrm{MW}}\right) / 2$. In the following, the normalised velocity will thus be defined as

$$
\beta=\frac{2 v_{\text {panto }}}{c_{\mathrm{CW}}+c_{\mathrm{MW}}}
$$




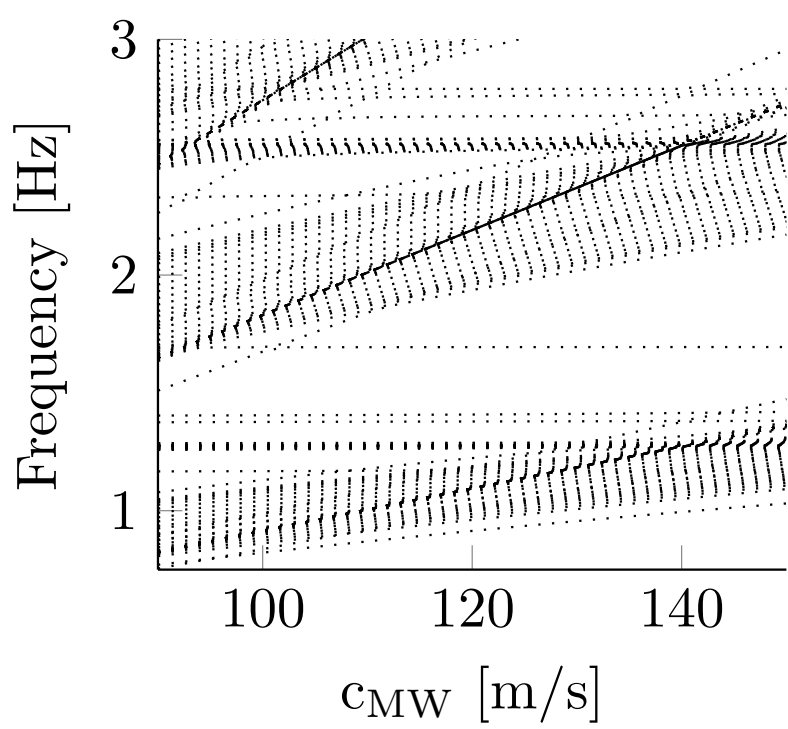

(a)

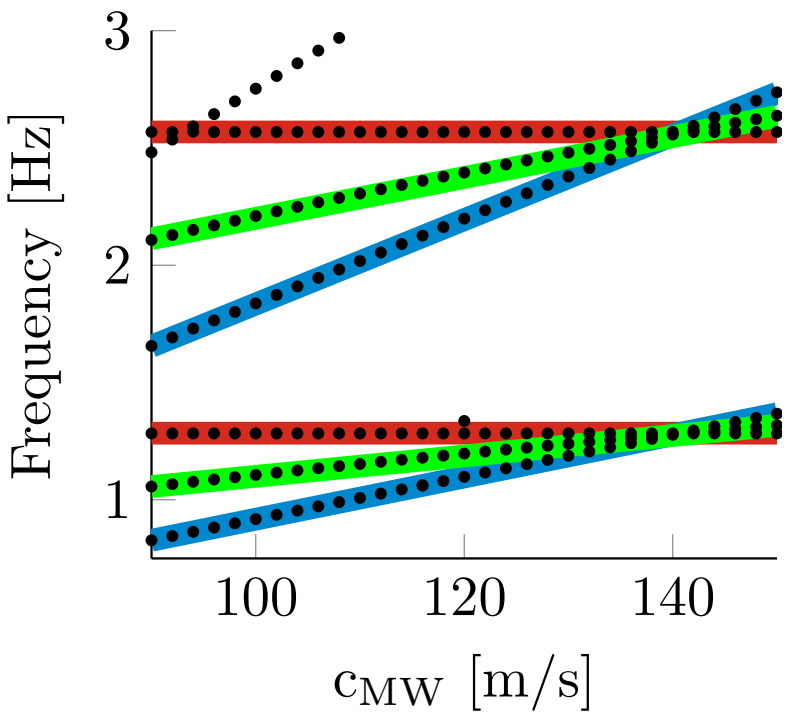

(b)

Figure 29: Frequency modes as a function of $c_{\mathrm{MW}}$. (a) Full catenary; (b) One 54m-length span.

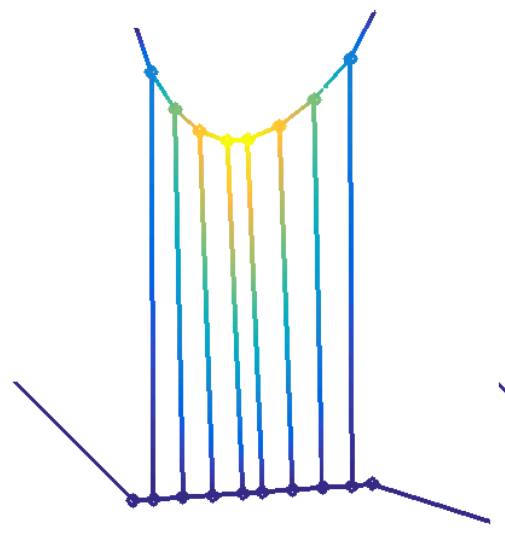

(a)

(b)

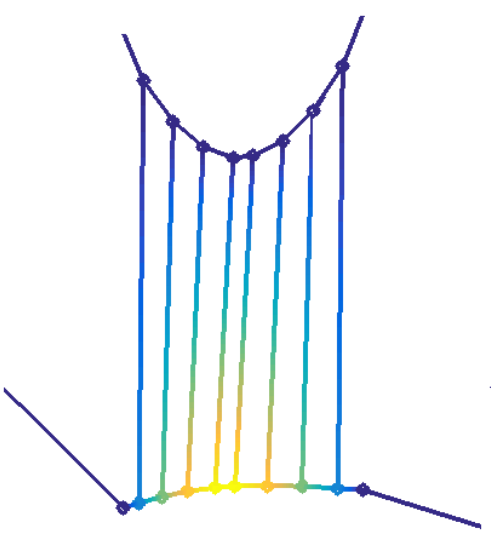

(c)

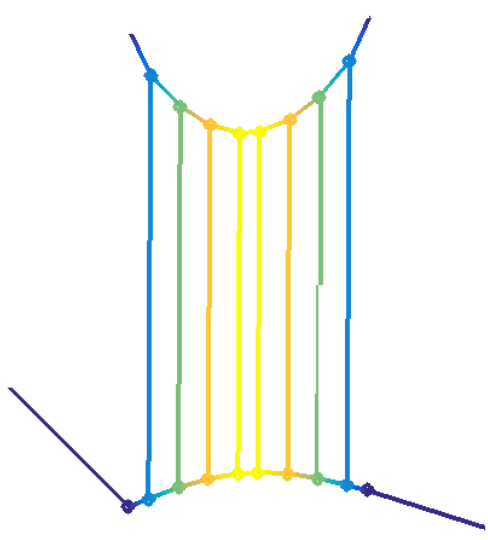

Figure 30: Span modes for $c_{\mathrm{MW}}=90 \mathrm{~m} / \mathrm{s}$ with (a) horizontal mode of $\mathrm{MW}$ at $0.89 \mathrm{~Hz}$; (b) horizontal mode of $\mathrm{CW}$ at $1.28 \mathrm{~Hz}$; (c) vertical coupled mode at $1.09 \mathrm{~Hz}$.

Figure 31a shows the evolution of the current collection quality criterion $\sigma / F_{m}$ as a function of the two parameters characterizing the messenger wire, namely $T_{\mathrm{MW}}$ and $\mu_{\mathrm{MW}}$. Variations observed are non monotonous and are thus difficult to exploit. Figure $31 \mathrm{~b}$ shows this same criterion as a function of the dynamic impedance of the messenger wire, $\sqrt{\mu_{\mathrm{MW}} T_{\mathrm{MW}}}$. Variations observed are monotonous and smooth. This confirms that couple defined in (23) is more adapted to study dynamic current collection quality. 


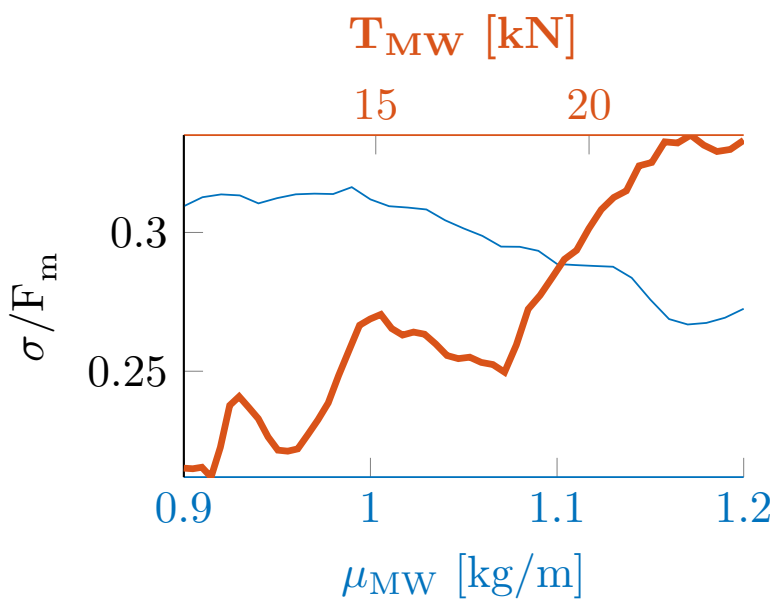

(a)

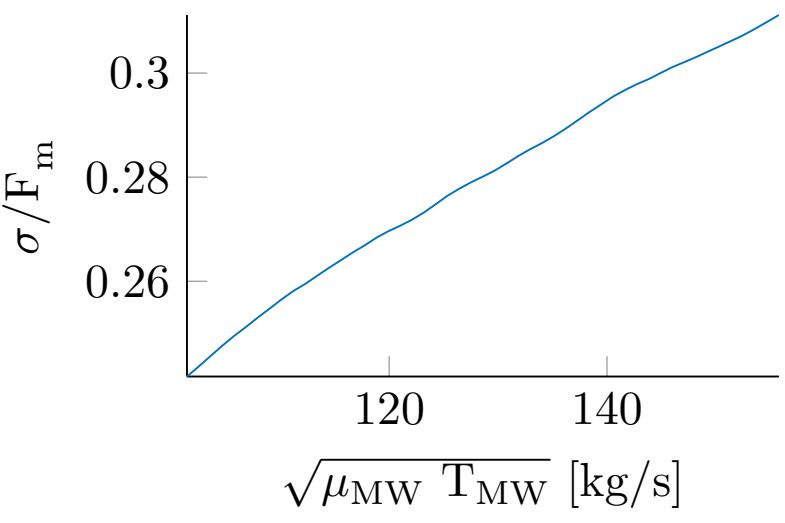

(b)

Figure 31: Variations of the current collection criterion $\sigma / F_{m}$. (a) as a function of $\mu_{\mathrm{MW}}$ (thin blue) and $T_{\mathrm{MW}}$ (thick red); (b) as a function of $\sqrt{\mu_{\mathrm{MW}} T_{\mathrm{MW}}}$

\section{Conclusions}

The first paper contribution was to give a priori information on most spectral components visible in the response and their dependence on velocity and measurement in the fixed frame associated with the catenary and in the moving frame associated with the pantograph. Identified sources of spectral components are the catenary geometry under gravity, pantograph impedance, time of flight of wave fronts and modes.

To analyze the wave front effects, analytical string models were also used to illustrate velocity compensation effects at droppers and masts. Experiments and FEM simulations showed that wave dispersion, present in beams, also adds significant higher frequency variations but that models are quite predictive.

Useful conclusions were drawn. First, waves generated at a dropper in the contact and messenger wires offset each other at the next or previous dropper. Then, at mast, waves in the messenger wire are fully reflected while reflections on steady arm are negligible. This behaviour confers a high importance to droppers surrounding the mast and to the ratio between dynamic impedances of wires.

The coincidence of frequencies or characteristic times were shown to explain most variations in the quantities of interest. In particular contact forces and uplift. For instance, if the pantograph velocity $v_{\text {panto }}$ is equal to $d_{\text {span }} f_{\text {mode }}$, then $v_{\text {panto }} k_{\text {span }}=f_{\text {mode }}$ and the current collection quality is significantly improved. Another coincidence is found for $f_{\text {wave,cat }}=f_{\text {mode }}$ and it was shown that the relevant wave velocity is the mean of wave velocities in contact and messenger wires.

Several observations described in this paper can be taken into account for catenary design. In particular, the reduction of the messenger wire dynamic impedance appeared to greatly improve current collection quality. New shapes or materials for messenger wire that can fulfil the electrical and mechanical requirements that have a smaller linear mass could thus be considered. The wave reflection in the messenger at mast also appeared to have its importance. Current collection could be improved if a way to attenuate the associated wave is found, for instance using a dissipative attachment. Finally, the influence of the first mode band on multi-pantograph performance was illustrated and opens perspectives for further robust design work.

\section{References}

[1] Y. H. Cho, K. Lee, Y. Park, B. Kang, K. N. Kim, Influence of contact wire pre-sag on the dynamics of pantograph railway catenary, International Journal of Mechanical Sciences 52 (11) (2010) 1471-1490. doi:10.1016/j.ijmecsci.2010.04.002. 
[2] M. Aboshi, M. Tsunemoto, Installation guidelines for Shinkansen high speed overhead contact lines, Quarterly Report of RTRI 52 (4) (2011) 230-236.

[3] J. Náprstek, Dynamic response of an overhead contact wire and pantograph collector to high speed moving load, in: G. Schmid, N. Chouw (Eds.), Proceedings of Wave 2000 Conference, 2000, pp. 121-130.

[4] A. Kumaniecka, Dynamics of the catenary modelled by a periodical structure, Journal of Theoretical and Applied Mechanics (2008) 869-878.

[5] M. Aboshi, K. Manabe, Analyses of contact force fluctuation between catenary and pantograph., Quarterly Report of RTRI 41 (3) (2000) 182-187. doi:10.2219/rtriqr.41.182.

[6] G. Bucca, A. Collina, A procedure for the wear prediction of collector strip and contact wire in pantograph-catenary system, Wear 266 (2009) 46-59. doi:10.1016/j.wear.2008.05.006.

[7] T. Usuda, Estimation of wear and strain of contact wire using contact force of pantograph, Quarterly Report of RTRI 48 (2007) 170-175. doi:10.2219/rtriqr.48.170.

[8] T. Koyama, Formation mechanism of undulating wear on overhead conductor rails due to dynamic characteristics of pantographs, Quarterly Report of RTRI 54 (1) (2013) 18-23.

[9] J.-P. Massat, E. Balmes, J.-P. Bianchi, Use of FEM models to study fatigue of overhead contact wire, in: Proceedings of Railways, 2016, pp. 1-20.

[10] A. Bobillot, J. P. Massat, J. P. Mentel, Design of pantograph-catenary systems by simulation, in: Proceedings of WCRR, 2011, pp. 1-13. doi:10.4203/ccp.93.2.

[11] Z. Liu, P.-A. Jönsson, S. Stichel, A. Rønnquist, On the implementation of an auxiliary pantograph for speed increase on existing lines, Vehicle System Dynamics 54 (8) (2016) 1077-1097. doi:10.1080/00423114.2016.1187278.

[12] T. J. Park, C. S. Han, J. H. Jang, Dynamic sensitivity analysis for the pantograph of a high-speed rail vehicle, Journal of Sound and Vibration 266 (2) (2003) 235-260. doi:10.1016/S0022-460X(02)01280-4.

[13] J. W. Kim, H. C. Chae, B. S. Park, S. Y. Lee, C. S. Han, J. H. Jang, State sensitivity analysis of the pantograph system for a high-speed rail vehicle considering span length and static uplift force, Journal of Sound and Vibration 303 (3-5) (2007) 405-427. doi:10.1016/j.jsv.2006.06.073.

[14] Y. Song, Z. Liu, H. Wang, X. Lu, J. Zhang, Nonlinear analysis of wind-induced vibration of high-speed railway catenary and its influence on pantograph catenary interaction, Vehicle System Dynamics 54 (6) (2016) $723-747$. doi:10.1080/00423114.2016.1156134.

[15] N. Zhou, W. Zhang, Investigation on dynamic performance and parameter optimization design of pantograph and catenary system, Finite Elements in Analysis and Design 47 (3) (2011) 288-295. doi:10.1016/j.finel.2010.10.008.

[16] S. Bruni, J. Ambrosio, A. Carnicero, Y. H. Cho, L. Finner, M. Ikeda, S. Y. Kwon, J.-P. Massat, S. Stichel, M. Tur, W. Zhang, The results of the pantograph - catenary interaction benchmark, Vehicle System Dynamics 53 (2) (2014) 1-24. doi:10.1080/00423114.2014.953183.

[17] Y. Song, Z. Liu, H. Wang, X. Lu, J. Zhang, Nonlinear modelling of high-speed catenary based on analytical expressions of cable and truss elements, Vehicle System Dynamics 53 (10) (2015) 1455-1479. doi:10.1080/00423114.2015.1051548.

[18] K. Lee, J. Chung, Dynamic analysis of a hanger-supported beam with a moving oscillator, Journal of Sound and Vibration 332 (13) (2013) 3177-3189. doi:10.1016/j.jsv.2013.01.015.

[19] J.-P. Massat, Modélisation du comportement dynamique du couple pantographe-caténaire. Application à la détection de défauts dans la caténaire [Modeling the dynamic behavior of the pantograph/catenary couple. Application to the detection of catenary defects], Ph.D. thesis, Ecole centrale de Lyon (2007).

[20] J.-P. Massat, E. Balmes, J.-P. Bianchi, G. Van Kalsbeek, OSCAR statement of methods, Vehicle System Dynamics 53 (3) (2015) 370-379. doi:10.1080/00423114.2015.1005016.

[21] O. Vo Van, E. Balmes, X. Lorang, Damping characterization of a high speed train catenary, in: International Symposium on Dynamics of Vehicles on Roads and Tracks, 2015. doi:10.1201/b21185-159.

[22] M. Géradin, D. J. Rixen, Mechanical Vibrations: Theory and Application to Structural Dynamics, 3rd Edition, Wiley, 2015.

[23] P. M. Morse, Vibration and Sound, 2nd Edition, McGraw-Hill, 1948. doi:10.1126/science.85.2211.479-a. 